\title{
Phosphatidylinositol 3,4-bisphosphate regulates neurite initiation and dendrite morphogenesis via actin aggregation
}

\author{
Shu-Xin Zhang ${ }^{1,2,{ }^{*}}$, Li-Hui Duan ${ }^{1,2, *}$, Shun-Ji He ${ }^{1}$, Gui-Feng Zhuang ${ }^{1}$, Xiang Yu ${ }^{1,2}$ \\ ${ }^{1}$ Institute of Neuroscience and State Key Laboratory of Neuroscience, CAS Center for Excellence in Brain Science and Intelligence \\ Technology, Chinese Academy of Sciences, Shanghai 200031, China; ${ }^{2}$ University of Chinese Academy of Sciences, Beijing 100049 , \\ China
}

Neurite initiation is critical for neuronal morphogenesis and early neural circuit development. Recent studies showed that local actin aggregation underneath the cell membrane determined the site of neurite initiation. An immediately arising question is what signaling mechanism initiated actin aggregation. Here we demonstrate that local clustering of phosphatidylinositol 3,4-bisphosphate $\left(\mathrm{PI}(3,4) \mathrm{P}_{2}\right)$, a phospholipid with relatively few known signaling functions, is necessary and sufficient for aggregating actin and promoting neuritogenesis. In contrast, the related and more extensively studied phosphatidylinositol 4,5-bisphosphate or phosphatidylinositol $(3,4,5)$-trisphosphate $\left(\mathrm{PIP}_{3}\right)$ molecules did not have such functions. Specifically, we showed that beads coated with $\mathrm{PI}(3,4) \mathrm{P}_{2}$ promoted actin aggregation and neurite initiation, while pharmacological interference with $\mathrm{PI}(3,4) \mathrm{P}_{2}$ synthesis inhibited both processes. $\mathrm{PI}(3,4) \mathrm{P}_{2}$ clustering occurred even when actin aggregation was pharmacologically blocked, demonstrating that $\mathrm{PI}(3,4) \mathrm{P}_{2}$ functioned as the upstream signaling molecule. Two enzymes critical for $\mathrm{PI}(3,4) \mathrm{P}_{2}$ generation, namely, SH2 domain-containing inositol 5-phosphatase and class II phosphoinositide 3-kinase $\alpha$, were complementarily and non-redundantly required for actin aggregation and neuritogenesis, as well as for subsequent dendritogenesis. Finally, we demonstrate that neural Wiskott-Aldrich syndrome protein and the Arp2/3 complex functioned downstream of $\mathrm{PI}(3,4) \mathrm{P}_{2}$ to mediate neuritogenesis and dendritogenesis. Together, our results identify $\mathrm{PI}(3,4) \mathrm{P}_{2}$ as an important signaling molecule during early development and demonstrate its critical role in regulating actin aggregation and neuritogenesis.

Keywords: PI(3,4) $\mathrm{P}_{2}$; actin aggregation; neuritogenesis; SHIP2; PI3K C2 $\alpha$; dendritogenesis; N-WASP

Cell Research (2017) 27:253-273. doi:10.1038/cr.2017.13; published online 20 January 2017

\section{Introduction}

Neurons are highly specialized cells with extensive axonal and dendritic arbors responsible for sending and receiving information, respectively. The morphological characteristics of these neuronal processes are critical for the ability of neuron to transmit and store information within the nervous system. Classical studies in cultured

\footnotetext{
*These two authors contributed equally to this work.

Correspondence: Xiang Yu

Tel: +86-21-54921827

E-mail: yuxiang@ion.ac.cn

Received 20 January 2016; revised 24 July 2016; accepted 19 October 2016; published online 20 January 2017
}

neurons have subdivided neuronal morphogenesis into five stages, including: (1) the formation of lamellipodial or filopodial processes from newborn neurons with spherical somata; (2) the extension of primary neurites from lamellipodia or filopodia; (3) the polarization of growing neurites into axons and dendrites; (4) the completion of axonal and dendritic arborizations; and (5) the establishment of synapses between the dendrites and axons of different neurons, and the formation of dendritic spines $[1,2]$. As compared to the last three stages of neuronal morphogenesis, which have been extensively studied [3-13], relatively little is known regarding the mechanism regulating neuritogenesis - the initial extension of neurites from the spherical soma [14-19]. Recent work from a number of laboratories, including our own, 
demonstrated a critical role of actin aggregation during neuritogenesis [20-23]. Using a combination of loss- and gain-of-function strategies, as well as pharmacological tools, these studies showed that the redistribution of evenly distributed cortical actin underneath the cell membrane into large actin aggregate(s) was required for neuritogenesis. Dynamics is key to this process, as actin bundling molecules, such as the Ena/Mena/VASP family of proteins (abbreviated as Ena/VASP), and actin depolymerizing factors, such as actin depolarizing factor (ADF)/Cofilin, have both been shown to be required for mediating local actin aggregation and subsequent neurite initiation from these sites [20-22]. In previous work, we subdivided stage 1 into two components, with neurons in stage 1a having evenly distributed cortical actin and those in stage $1 \mathrm{~b}$ having one or two large actin aggregates directly underneath the plasma membrane [23].

An immediate follow-up question is what upstream mechanism regulates actin aggregation. Previous work has shown that the extracellular guidance molecule Semaphorin 3A promotes formation of the apical dendrite of pyramidal neurons towards the pia surface of the cerebral cortex [24]. Brain-derived neurotrophic factor, another extracellular signaling molecule, has been shown to promote axon differentiation and dendrite growth $[25$, 26], while the homophilic adhesion molecule $\mathrm{N}$-cadherin has been shown to be asymmetrically distributed during early neuronal development [27]. In contrast, intrinsic mechanisms regulating neuritogenesis have been little explored. We surmise that intrinsic regulation of neuritogenesis is critical, as neurons have a limited number of primary neurites (one axon and multiple dendrites), and this number is essentially constant for each neuronal type [28]. Since actin aggregates form directly beneath the plasma membrane, we propose that their intrinsic upstream regulators likely have the following characteristics: (1) be membrane bound; (2) appear in localized manner prior to and/or concurrent with actin aggregates; and (3) be highly dynamic but limited in total amount. The first two requirements need no further explanation. For the third, since most neurons extend two neurites initially, and only have a total of 5-7 neurites in the mature state, this signaling mechanism only needs to be activated under rare and limited circumstances.

Focusing on membrane-bound intrinsic signals, our attention was drawn to phosphoinositides (PIs), phosphorylated derivatives of the phospholipid phosphatidylinositol. They are minor acidic phospholipids localized to the cytosolic surface of membrane and can be reversibly phosphorylated at positions 3,4 and/or 5 to generate a total of 7 possible derivatives [29-33]. Although PIs only account for a small proportion of total lipids in the membrane, they are important signaling molecules with diverse functions, including the regulation of membrane trafficking, intracellular signaling, cytoskeleton organization and apoptosis [29-33]. Of the PIs shown to be present in the plasma membrane, the most well characterized in terms of signal transduction are phosphatidylinositol 4,5-bisphosphate $\left(\mathrm{PI}(4,5) \mathrm{P}_{2}\right)$ and $\mathrm{PI}(3,4,5) \mathrm{P}_{3}\left(\mathrm{PIP}_{3}\right.$ for short) [29-34]. In neuronal morphogenesis, $\mathrm{PIP}_{3}$ and its upstream regulator class I PI3 kinase (PI3K) have previously been shown to regulate axon specification and neuronal polarization [35-37]. In epithelial cells, the localization of $\mathrm{PI}(4,5) \mathrm{P}_{2}$ and $\mathrm{PIP}_{3}$ to distinct subcellular compartments has been shown to be essential for the development and maintenance of epithelial polarity [38-40]. More recently, the related phosphatidylinositol 3,4-bisphosphate $\left(\mathrm{PI}(3,4) \mathrm{P}_{2}\right)$ has also been demonstrated to be more than just a byproduct of $\mathrm{PIP}_{3}$ degradation and rather an important signaling molecule of its own right [30, $32,34]$. All three PIs can regulate the organization and dynamics of the actin cytoskeleton, with $\mathrm{PI}(4,5) \mathrm{P}_{2}$ being the most extensively studied [29-31, 34].

Here we investigate the role of PIs in neurite initiation and demonstrate that local clustering of $\mathrm{PI}(3,4) \mathrm{P}_{2}$, but not $\mathrm{PI}(4,5) \mathrm{P}_{2}$ or $\mathrm{PIP}_{3}$, is spatially correlated with the formation of large actin aggregates underneath the plasma membrane and the determination of the neurite initiation site. Using pharmacological manipulations to interfere with the synthesis and degradation of PIs, as well as PI-coated beads to induce local increase in its concentration, we demonstrate a necessary and sufficient role of $\mathrm{PI}(3,4) \mathrm{P}_{2}$ in regulating actin aggregation and neuritogenesis. Further analyses using gain- and loss-of-function genetic manipulations identified the signaling pathways upstream and downstream of $\mathrm{PI}(3,4) \mathrm{P}_{2}$. The determinant role of $\mathrm{PI}(3,4) \mathrm{P}_{2}$ in regulating actin aggregation and neurite initiation further highlights the importance of PIs in early neuronal development, as defects in their metabolism have been associated with various developmental disorders [29, 32, 33].

\section{Results}

$P I(3,4) P_{2}$ co-localizes with actin aggregates during neuritogenesis

To determine whether PIs regulated actin aggregation and neuritogenesis, we first assayed whether $\mathrm{PIP}_{3}, \mathrm{PI}(3,4)$ $\mathrm{P}_{2}$ or $\mathrm{PI}(4,5) \mathrm{P}_{2}$ co-localized with actin aggregates during early neurite initiation. Cultured hippocampal neurons were fixed 3-6 $\mathrm{h}$ after plating, at a developmental stage where they typically had one or two large actin aggregates underneath the plasma membrane, defined as stage $1 \mathrm{~b}$ in our previous study [23] (Figure 1F). Neurons were 
A
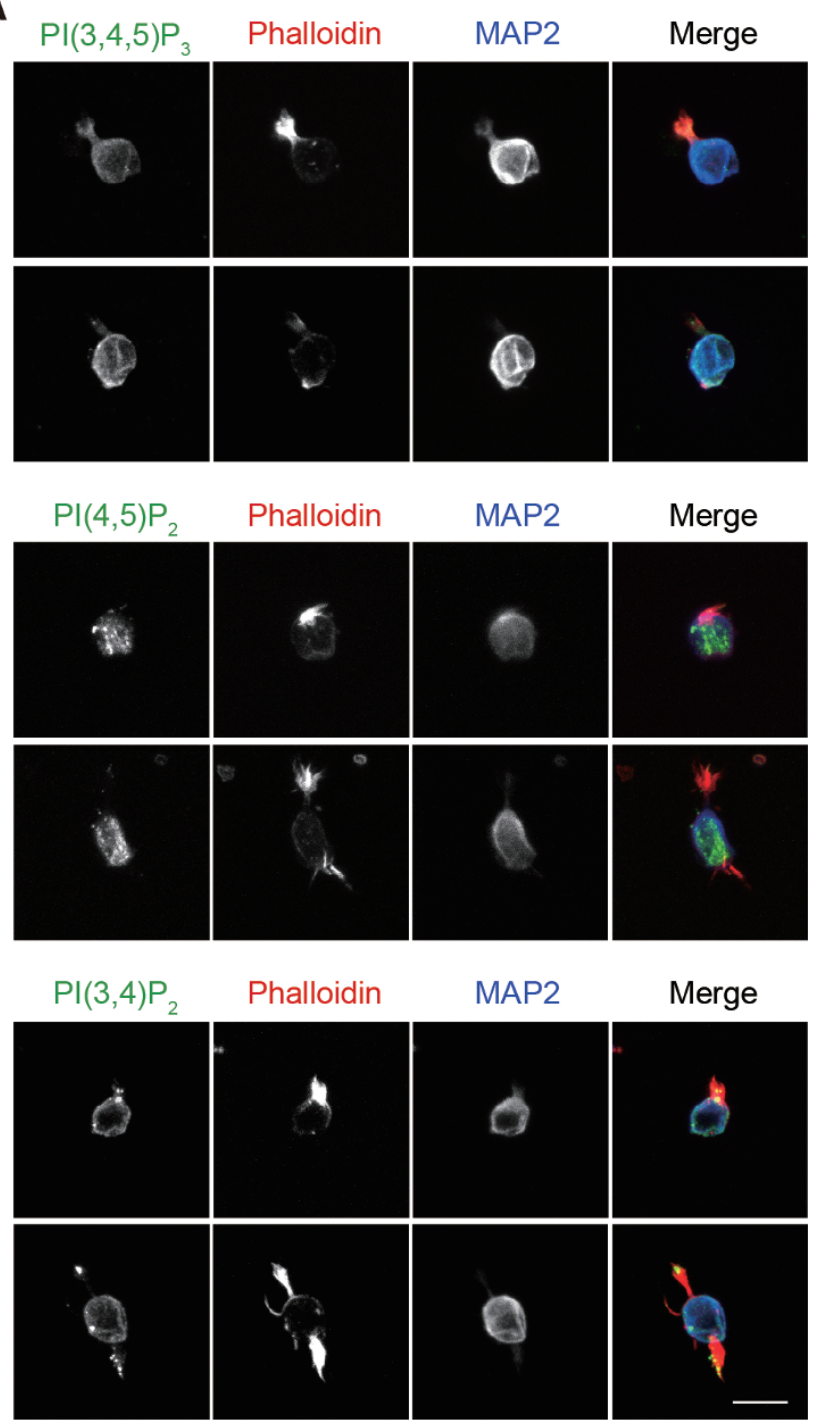

E

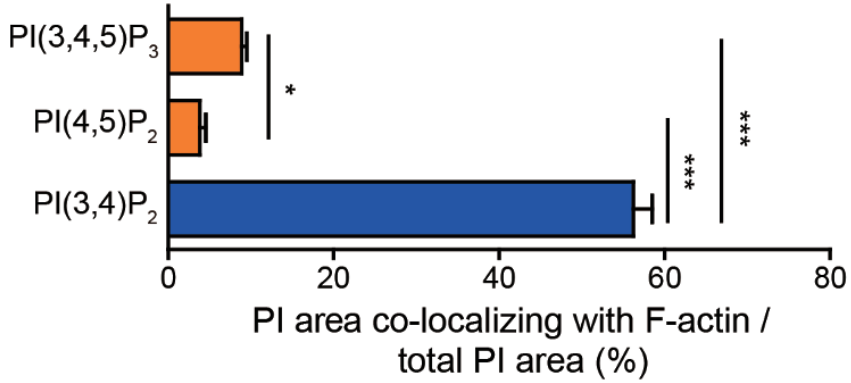

B

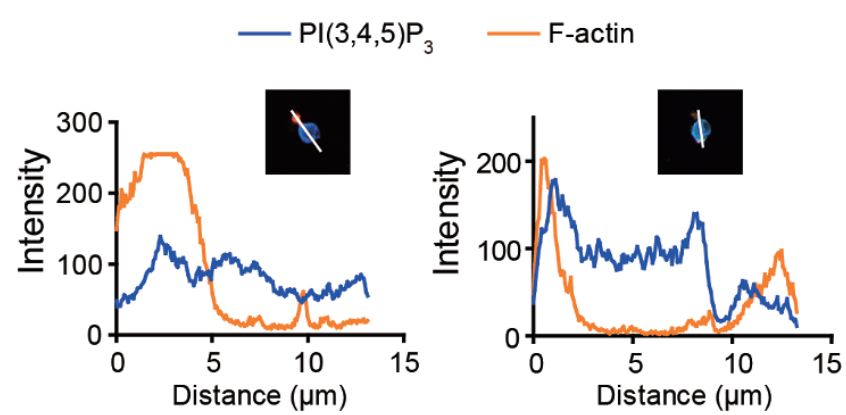

C
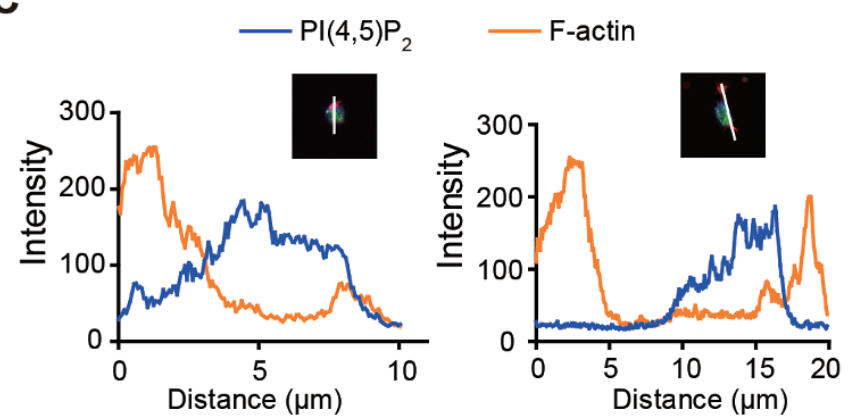

D
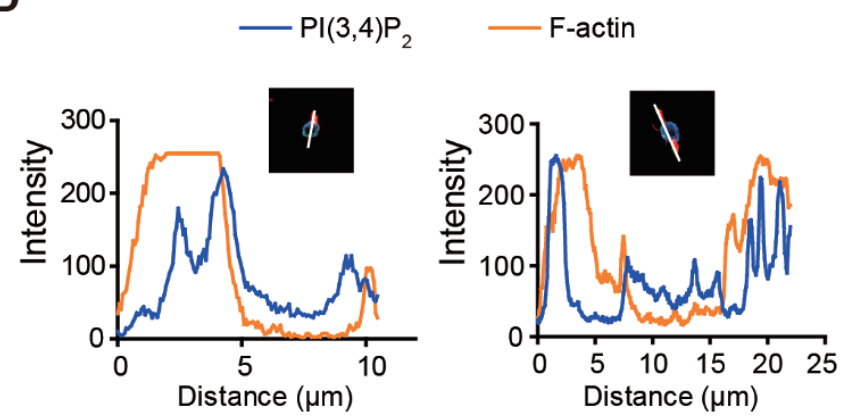

$\mathbf{F}$

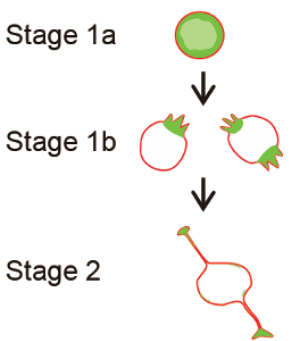

Figure $1 \mathrm{PI}(3,4) \mathrm{P}_{2}$ co-localizes with actin aggregates during neurite initiation. (A) Representative images of cultured rat hippocampal neurons fluorescently labeled for $\mathrm{PI}(3,4,5) \mathrm{P}_{3}, \mathrm{PI}(4,5) \mathrm{P}_{2}$ or $\mathrm{PI}(3,4) \mathrm{P}_{2}$ (green channel), F-actin (phalloidin, red channel) and MAP2 (blue channel). Scale bar, $10 \mu \mathrm{m}$. (B-D) Fluorescence intensity of $\mathrm{PI}$ and F-actin in example neurons shown in $\mathbf{A}$, measured along the white line indicated in the insets. (E) Quantification of percentage of co-localization between F-actin and $\mathrm{PI}(3,4,5) \mathrm{P}_{3}, \mathrm{PI}(4,5) \mathrm{P}_{2}$ or $\mathrm{PI}(3,4) \mathrm{P}_{2} ; n=123,120$ and 119 neurons, respectively; one-way ANOVA followed by Tukey's post hoc test. (F) Schematic of Stages 1a, $1 \mathrm{~b}$ and 2 of neuronal development, where green shading represents F-actin aggregates. In this and all subsequent figures, error bars represent SEM, and ${ }^{*} P<0.05$, ${ }^{* *} P<0.01,{ }^{* * *} P<0.001$, and n.s., not significant, as indicated on graphs. 
identified by immunostaining for the microtubule-associated protein 2 (MAP2), while filamentous actin (F-actin) was visualized using phalloidin. As shown in Figure 1A, although $\mathrm{PIP}_{3}$ and $\mathrm{PI}(4,5) \mathrm{P}_{2}$ were present at relatively high levels in the cell body of early developing neurons, they did not significantly overlap with phalloidin staining. In contrast, the distribution of $\mathrm{PI}(3,4) \mathrm{P}_{2}$ was very punctate, and overlapped significantly with that of phalloidin (Figure 1A).

We further quantified the fluorescence intensity of F-actin and the PIs by transecting the cell body and its actin aggregates with a straight line and measuring fluorescent intensity of both parameters along the line. For the three PIs examined, only the intensity of $\mathrm{PI}(3,4) \mathrm{P}_{2}$ significantly overlapped with that of F-actin along the line (Figure 1B-1D). Furthermore, $56.22 \% \pm 2.29 \%$ of the $\mathrm{PI}(3,4) \mathrm{P}_{2}$ signal co-localized with the F-actin signal, whereas the same measurements for $\mathrm{PIP}_{3}$ or $\mathrm{PI}(4,5) \mathrm{P}_{2}$ were $8.89 \% \pm 0.64 \%$ and $3.81 \% \pm 0.73 \%$, respectively (Figure 1E). Together, these results suggest that PI $(3,4)$ $\mathrm{P}_{2}$, the least previously studied PI, co-localized with actin aggregates during the early stage of neuronal morphogenesis.

$P I(3,4) P_{2}$ is necessary and sufficient for actin aggregation and neurite initiation

To further investigate whether any of the PIs were required for actin aggregation, we treated cultured hippocampal neurons with pharmacological agents that inhibited the synthesis of $\mathrm{PI}(3,4) \mathrm{P}_{2}, \mathrm{PI}(4,5) \mathrm{P}_{2}$ or $\mathrm{PIP}_{3}$. As shown in Figure 2A, these included: (1) LY294002, an inhibitor of class I PI3K-mediated conversion of PI $(4,5)$ $\mathrm{P}_{2}$ to $\mathrm{PIP}_{3}$; (2) $m$-3M3FBs, an activator of phospholipase C (PLC)-mediated conversion of $\mathrm{PI}(4,5) \mathrm{P}_{2}$ to diacylglycerol (DAG) and inositol trisphosphate ( $\left.\mathrm{IP}_{3}\right)$; and (3) AS1949490, an inhibitor of SH2 domain-containing inositol 5-phosphatase (SHIP2)-mediated conversion of PIP $_{3}$ to $\mathrm{PI}(3,4) \mathrm{P}_{2}$. Drugs were added to cultured hippocampal neurons at the time of plating using concentrations previously reported to fully inhibit the function of these enzymes [41-43], and their effects were examined $6 \mathrm{~h}$ post plating. Neurons were identified by immunostaining for MAP2 or for the neuron-specific class III $\beta$-tubulin, Tuj 1 . The specificities of the pharmacological agents and the antibodies were first confirmed by immunostaining for each PI in the presence of each pharmacological agent in all possible permutations (Supplementary information, Figure S1). In terms of neuronal morphogenesis, treatment with neither LY294002 $(100 \mu \mathrm{M})$ nor $m$-3M3FBs $(50 \mu \mathrm{M})$ significantly affected the formation of actin aggregates (Figure 2B), although they both slightly delayed the progression of neuronal development from stage 1a to stage 2 (Figure 2B and 2C). In contrast, AS1949490 $(70 \mu \mathrm{M})$ nearly abolished the formation of actin aggregates and stalled $64.45 \% \pm 4.78 \%$ of neurons at stage $1 \mathrm{a}$, with uniform F-actin underneath the cell membrane, as compared with only $5.83 \% \pm 1.19 \%$ of neurons in stage 1a under control conditions (Figure 2B and 2C). In terms of the total number of neurites extending from the cell body, all three treatments significantly reduced neurite number, with the effects of AS1949490 being the most striking (Figure 2D).

Although each of the above-described pharmacological agents, at the concentration used, specifically reduced the level of the correspondent PI (Supplementary information, Figure S1), since PIs can be interconverted from each other, high concentration of a broad spectrum PI3K inhibitor, such as wortmannin, is also expected to block neuronal morphogenesis at high concentrations. Indeed, while $1 \mu \mathrm{M}$ wortmannin had no significant effects, 10 $\mu \mathrm{M}$ and $50 \mu \mathrm{M}$ wortmannin significantly delayed neuronal morphogenesis and reduced neurite number (Supplementary information, Figure S2A-S2C). Consistently, 50 $\mu \mathrm{M}$ wortmannin almost completely abolished $\mathrm{PI}(3,4) \mathrm{P}_{2}$ clustering (Supplementary information, Figure S2D and S2E).

In addition to demonstrating that AS1949490 specifically inhibited the production of $\mathrm{PI}(3,4) \mathrm{P}_{2}$, and that $\mathrm{PI}(3,4) \mathrm{P}_{2}$ co-localized with F-actin, it is also important to ask whether $\mathrm{PI}(3,4) \mathrm{P}_{2}$ is sufficient for inducing neuronal morphogenesis. To locally increase the concentration of $\mathrm{PI}(3,4) \mathrm{P}_{2}$, we combined the use of polybasic carrier protein to deliver PIs into intracellular membranes [44], and the ability of glass beads to locally increase the concentration of coated molecules $[45,46]$. When newly plated cultured neurons were treated with $\mathrm{PI}(3,4) \mathrm{P}_{2}$-coated glass beads for $3 \mathrm{~h}, 60.98 \% \pm 2.05 \%$ of all $\mathrm{PI}(3,4) \mathrm{P}_{2}$-coated beads in direct contact with cell bodies co-localized with an actin aggregate or neurite (Figure $2 \mathrm{E}$ and $2 \mathrm{~F}$ ). This was in sharp contrast to treatment with carrier-coated beads or $\mathrm{PIP}_{3}$-coated beads, where co-localization between beads and actin aggregates/neurites were $37.94 \%$ $\pm 3.23 \%$ and $31.25 \% \pm 5.00 \%$, respectively (Figure 2E and $2 \mathrm{~F}$ ). Together, these results demonstrate that $\mathrm{PI}(3,4)$ $\mathrm{P}_{2}$ is both necessary and sufficient for inducing local actin aggregation and neurite initiation.

\section{$P I(3,4) P_{2}$ clustering occurs even if F-actin does not re- distribute}

A final criterion for $\mathrm{PI}(3,4) \mathrm{P}_{2}$ to function as the upstream regulator of actin aggregation and neurite initiation, is that it is localized at punctate structures, even when $\mathrm{F}$-actin reorganization and neuritogenesis are blocked. As we previously reported, this can be achieved 
A

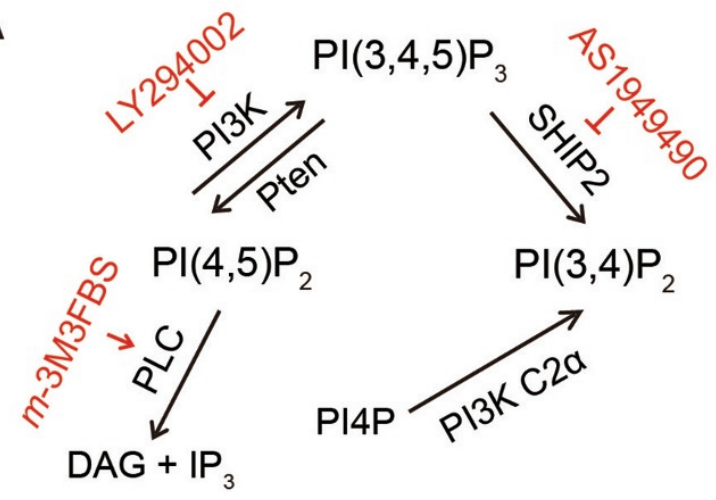

B

Phalloidin
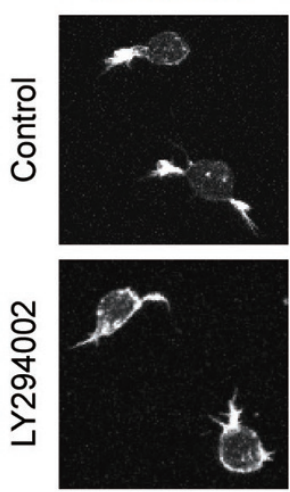

Tuj 1
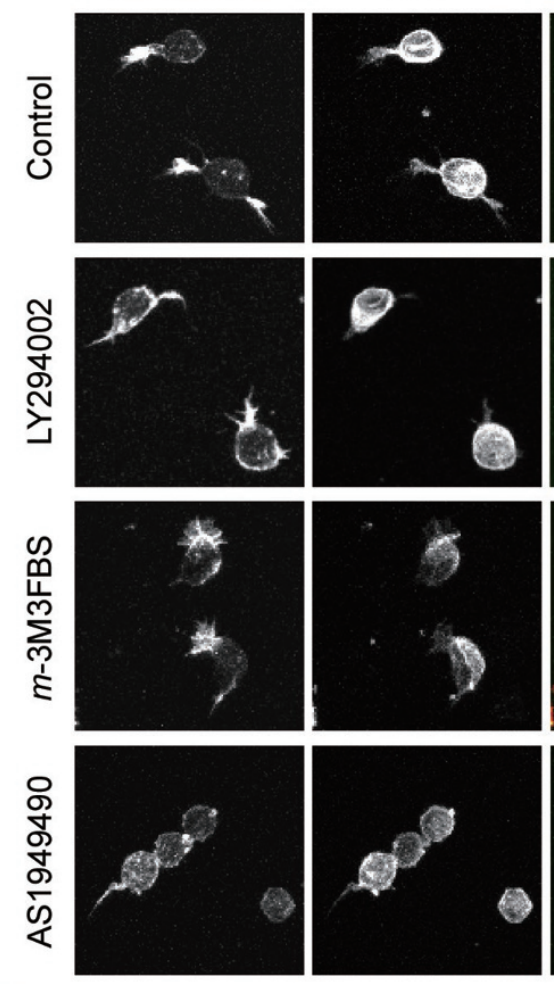

E
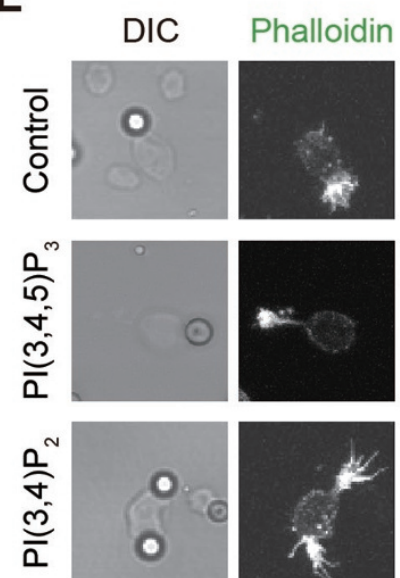

Merge
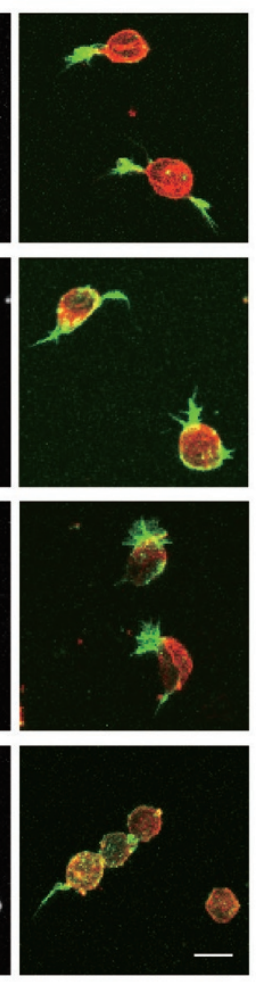

Tuj 1 Merge + DIC
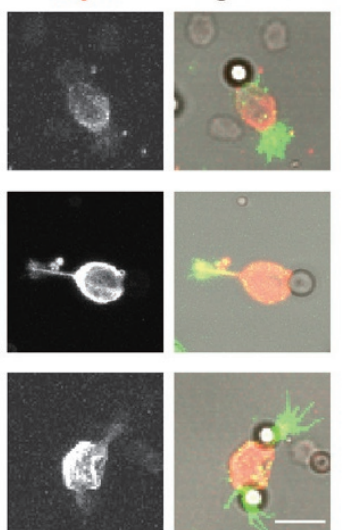

C
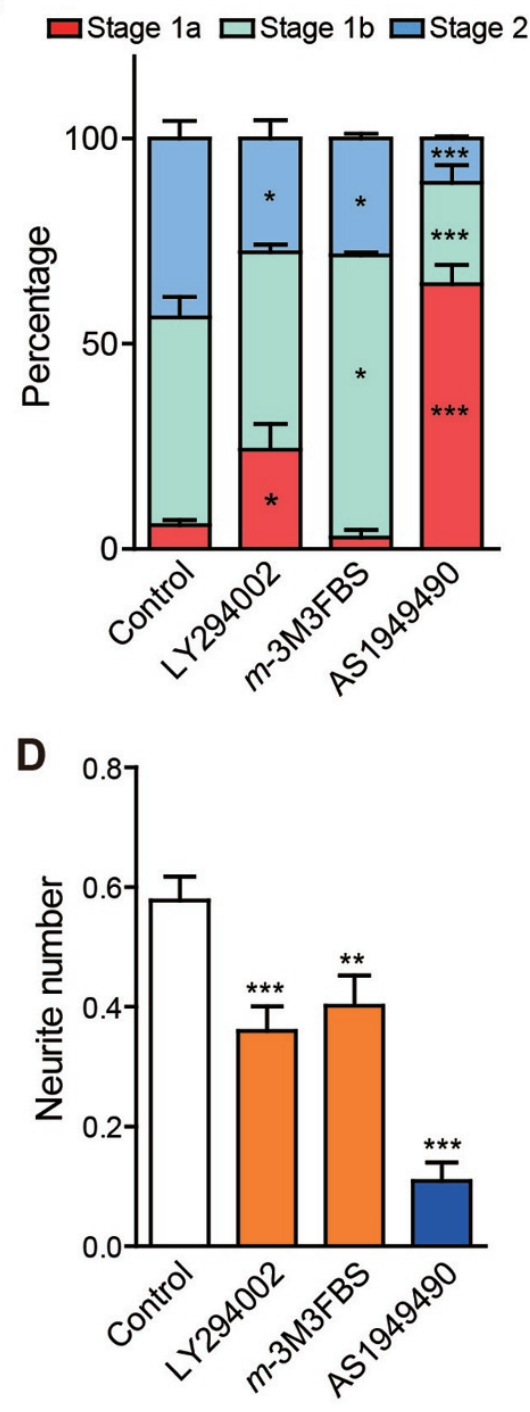

F

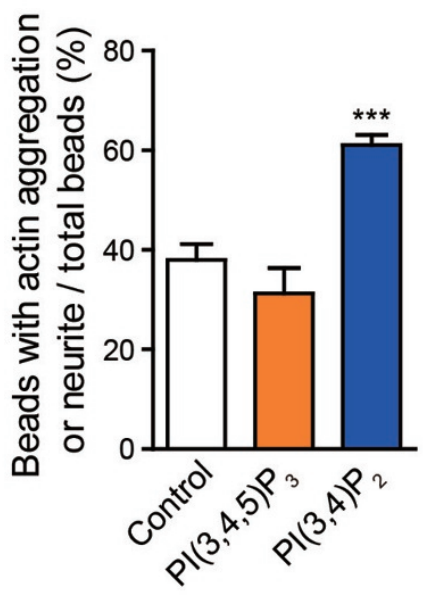


Figure $2 \mathrm{PI}(3,4) \mathrm{P}_{2}$ is necessary and sufficient for inducing actin aggregation and neurite initiation. (A) Schematic showing the enzymes responsible for interconversion between PIs, as well as the pharmacological agents used in this study (shown in red). (B) Representative images of neurons treated with vehicle DMSO (Control), $100 \mu \mathrm{M}$ LY294002, $50 \mu \mathrm{M} \mathrm{m-3M3FBS} \mathrm{or} 70$ $\mu \mathrm{M}$ AS1949490. Neurons were labeled for phalloidin (green) and Tuj 1 (red). (C, D) Quantification of developmental stages (C) and neurite number (D). $n=374,228,214$ and 101 neurons, respectively; two-way ANOVA followed by Bonferroni's post hoc test in C, and one-way ANOVA followed by Dunnett's post hoc test in D. (E) Representative images of neurons treated with beads coated with carrier alone (control), carrier and $\mathrm{PI}(3,4,5) \mathrm{P}_{2}$ or carrier and $\mathrm{PI}(3,4) \mathrm{P}_{2}$. Neurons were labeled for phalloidin (green) and Tuj 1 (red); beads were visualized under DIC. (F) Quantification of neuritogenesis and actin aggregation. A total of 463 beads from 70 image frames were quantified in the control group, 108 beads from 40 frames in the $\mathrm{PI}(3,4,5) \mathrm{P}_{3}$ group and 382 beads from 30 frames in the $\mathrm{PI}(3,4) \mathrm{P}_{2}$ group; one-way ANOVA followed by Tukey's post hoc test. Scale bar, $10 \mu \mathrm{m}$.

through treatment with the F-actin-stabilizing agent jasplakinolide (100 nM) [23]. Cultured neurons treated with jasplakinolide were immunostained for $\mathrm{PI}(3,4) \mathrm{P}_{2}$ at 0.5 , 3 and $6 \mathrm{~h}$ after plating, and their developmental stages were compared with control neurons. At both 3 and $6 \mathrm{~h}$ post plating, jasplakinolide-treated neurons were mostly stalled in stage 1a, with evenly distributed F-actin underneath the cell membrane, as compared to control neurons that have progressed to stages $1 \mathrm{~b}$ or stage 2 , respectively, with F-actin clusters at end(s) of the cell body or extending neurites (Figure 3A and 3B). Jasplakinolide-treated neurons also had significantly fewer neurites extending from the cell body at $6 \mathrm{~h}$ post plating (Figure 3C). Importantly, despite significant defects in both actin aggregate formation and neurite initiation, the intensity of $\mathrm{PI}(3,4) \mathrm{P}_{2}$ puncta was not affected by jasplakinolide treatment at 3 $h$ (Figure 3D). By 6 h, the PI $(3,4) \mathrm{P}_{2}$ signal was still highly punctate in jasplakinolide-treated neurons, although its intensity was lower than that of control neurons (Figure 3D). These results clearly demonstrate that $\operatorname{PI}(3,4)$ $\mathrm{P}_{2}$ clustering occurs in the absence of actin aggregation and it functions upstream of F-actin aggregate formation. The $6 \mathrm{~h}$ results also suggest a potential positive feedback loop between F-actin and $\mathrm{PI}(3,4) \mathrm{P}_{2}$ to further amplify the initial signal to aggregate (see the Discussion section).

\section{Regulation of PI $(3,4) P_{2}$ level by SHIP 2 and PI3K C2 $\alpha$}

Having determined a critical role for $\mathrm{PI}(3,4) \mathrm{P}_{2}$ in regulating actin aggregation and neurite initiation, we next asked how endogenous $\mathrm{PI}(3,4) \mathrm{P}_{2}$ level was regulated. $\mathrm{PI}(3,4) \mathrm{P}_{2}$ can be generated either by the dephosphorylation of $\mathrm{PI}(3,4,5) \mathrm{P}_{3}$ at the 5 th position by phosphatases SHIP1 and SHIP2 or by the phosphorylation of phosphatidylinositol 4-phosphate (PI(4)P) at the 3rd position by class II PI3K [29-32]. For the phosphatases, since SHIP1 is only expressed in hematopoietic cells [47], we focused on SHIP2, which is expressed in all cells and especially at high levels in the ventricular zones and subventricular zones of the mouse embryo and in adult stem cells [4850], indicating a role in regulating early neuronal development. Class II PI3Ks include class II phosphoinositide 3-kinase alpha (PI3K C2 $\alpha$ ), PI3K C2 $\beta$ and PI3K C2 $\gamma$, with $\mathrm{C} 2 \alpha$ and $\mathrm{C} 2 \beta$ isoforms being ubiquitously expressed [51-53]. Since loss of function of PI3K C $2 \alpha$ led to early embryonic lethality $[53,54]$, demonstrating that it has a non-redundant role in development, we focused on PI3K $\mathrm{C} 2 \alpha[54,55] . \mathrm{PI}(3,4) \mathrm{P}_{2}$ puncta were clearly detectable in neurons $3 \mathrm{~h}$ post plating, thus the endogenous expression of SHIP2 or PI3K C2 $\alpha$ needed to be manipulated at an earlier time point. We achieved this by in utero electroporating rat embryos at E15.5, using either SHIP2 RNAi [56] or PI3K C2 $\alpha$ RNAi construct [57], together with GFP to visualize the electroporated neurons. The efficiency and specificity of the RNAi constructs were confirmed using both western blot and immunocytochemistry (Supplementary information, Figure S3A-S3F). Cortical neurons were then cultured from E18.5 embryos and labeled using phalloidin and Tuj 1 antibody. As compared to control neurons from the same culture preparations, $\operatorname{PI}(3,4)$ $\mathrm{P}_{2}$ level in neurons expressing SHIP2 RNAi or PI3K C2 $\alpha$ RNAi construct was significantly reduced (Figure 4A4D). Furthermore, these neurons were developmentally delayed, as measured by more neurons stalled in stage 1a and significantly fewer neurites in neurons (Figure 4E$4 \mathrm{G})$.

In an alternative assay for measuring neurite number, cortices electroporated with SHIP2 RNAi or PI3K C2 $\alpha$ RNAi construct at E15.5, together with the red fluorescent protein tdTomato to mark neuronal morphology, were fixed at E18.5. As compared to neurons electroporated with control constructs, those with reduced endogenous level of either protein had fewer neurites, as demonstrated by a significant increase in the proportion of neurons with no neurites and a significant reduction in the proportion of neurons with two neurites (Figure 5). Together, these results demonstrate that both SHIP2 and PI3K C2 $\alpha$ contributed significantly to $\mathrm{PI}(3,4) \mathrm{P}_{2}$-dependent actin aggregation and neuritogenesis.

If, as we proposed, the main function of SHIP2 and PI3K C $2 \alpha$ was to locally increase PI $(3,4) \mathrm{P}_{2}$ level, we would expect PI $(3,4) \mathrm{P}_{2}$-coated beads to rescue the effects of SHIP2 RNAi or PI3K C2 $\alpha$ RNAi. Indeed, when neu- 
A

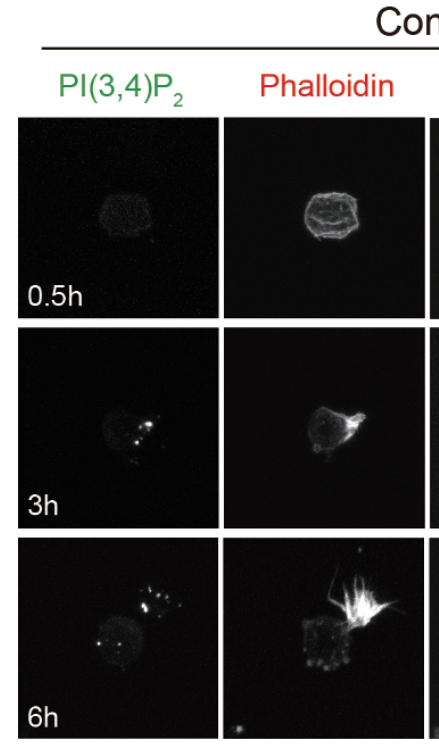

B

$\square$ Stage 1a $\square$ Stage 1b $\square$ Stage 2

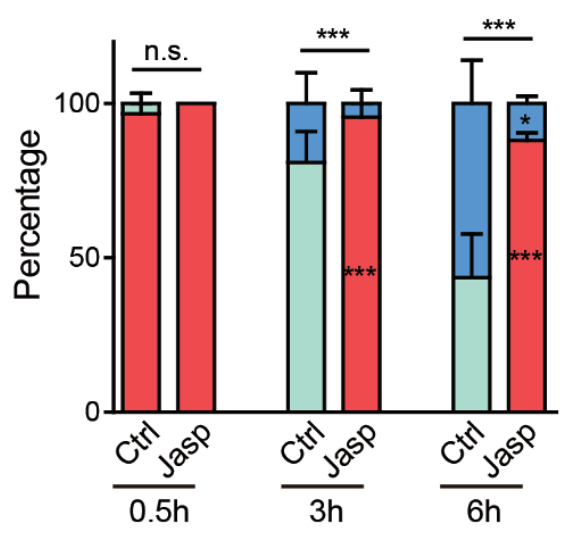

C

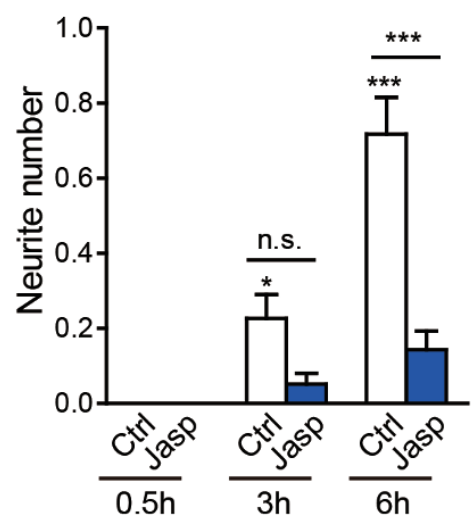

Jasplakinolide (Jasp)
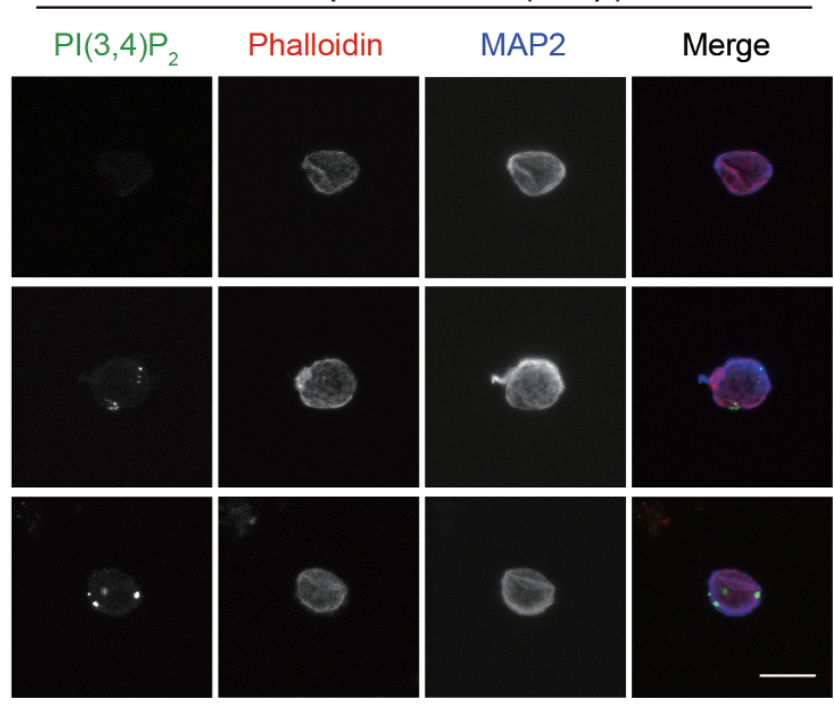

D

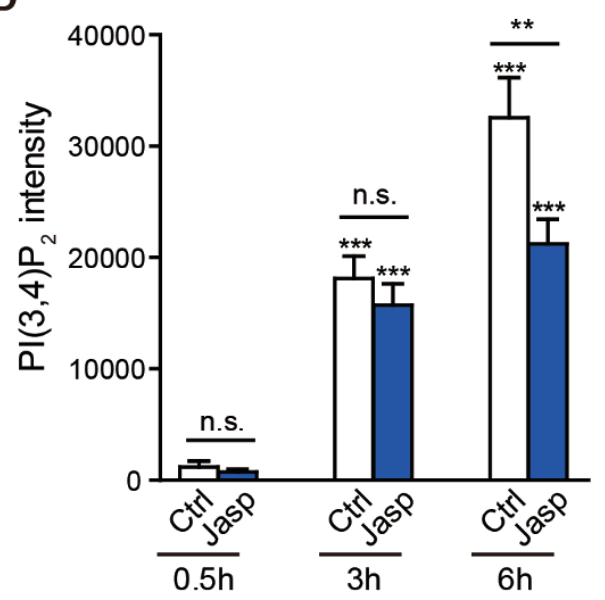

Figure $3 \mathrm{PI}(3,4) \mathrm{P}_{2}$ clusters appear independent of actin aggregation or neurite initiation. (A) Representative images of neurons labeled for $\mathrm{PI}(3,4) \mathrm{P}_{2}$ (green), phalloidin (red) and MAP2 (blue), and treated with DMSO (control) or $100 \mathrm{nM}$ Jasplakinolide for $0.5,3$ or $6 \mathrm{~h}$. Scale bar, $10 \mu \mathrm{m}$. (B-D) Quantification of neuronal developmental stages (B), neurite number (C) and $\mathrm{PI}(3,4) \mathrm{P}_{2}$ intensity (D). $n=52,53$ and 53 neurons for the control groups, and 50, 58 and 63 neurons for the jasplakinolide groups for each time point. Two-way ANOVA followed by Bonferroni's post hoc test was used for comparing condition pairs at each time point in $\mathbf{B},{ }^{* * *} P<0.001$ for stage $1 \mathrm{~b}$ at 3 and $6 \mathrm{~h}$ time points; one-way ANOVA followed by Tukey's post hoc test was used in $\mathbf{C}$ and $\mathbf{D}$.

rons in utero electroporated with SHIP2 RNAi or PI3K $\mathrm{C} 2 \alpha$ RNAi construct were treated with $\mathrm{PI}(3,4) \mathrm{P}_{2}$-coated beads, actin aggregate formation and/or neurite initiation was significantly enhanced, as compared to neurons treated with control beads or $\mathrm{PIP}_{3}$-coated beads (Figure 6).

\section{SHIP 2 and PI3K C2 $\alpha$ are non-redundant during neurito- genesis}

Each neuron has a single axon and multiple dendrites. Do SHIP2 and PI3K C2 $\alpha$ affect the development of both axons and dendrites? To examine this, we electroporated hippocampal neurons at the time of plating with SHIP2 RNAi or PI3K C2 $\alpha$ RNAi construct, together with GFP to mark neuronal morphology. Neurons were fixed for analysis at days in vitro (DIV) 3. By this stage, axons can be clearly distinguished by their longer length and even diameter throughout their arbors. Examining axonal and dendritic development separately, we found that both RNAi conditions significantly reduced the number of primary dendrites, as well as total dendrite length (Fig- 
A
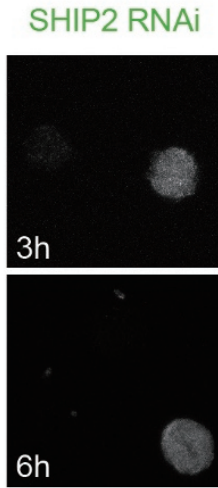

C

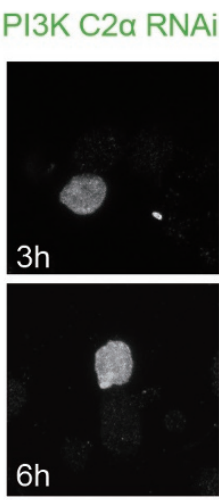

E
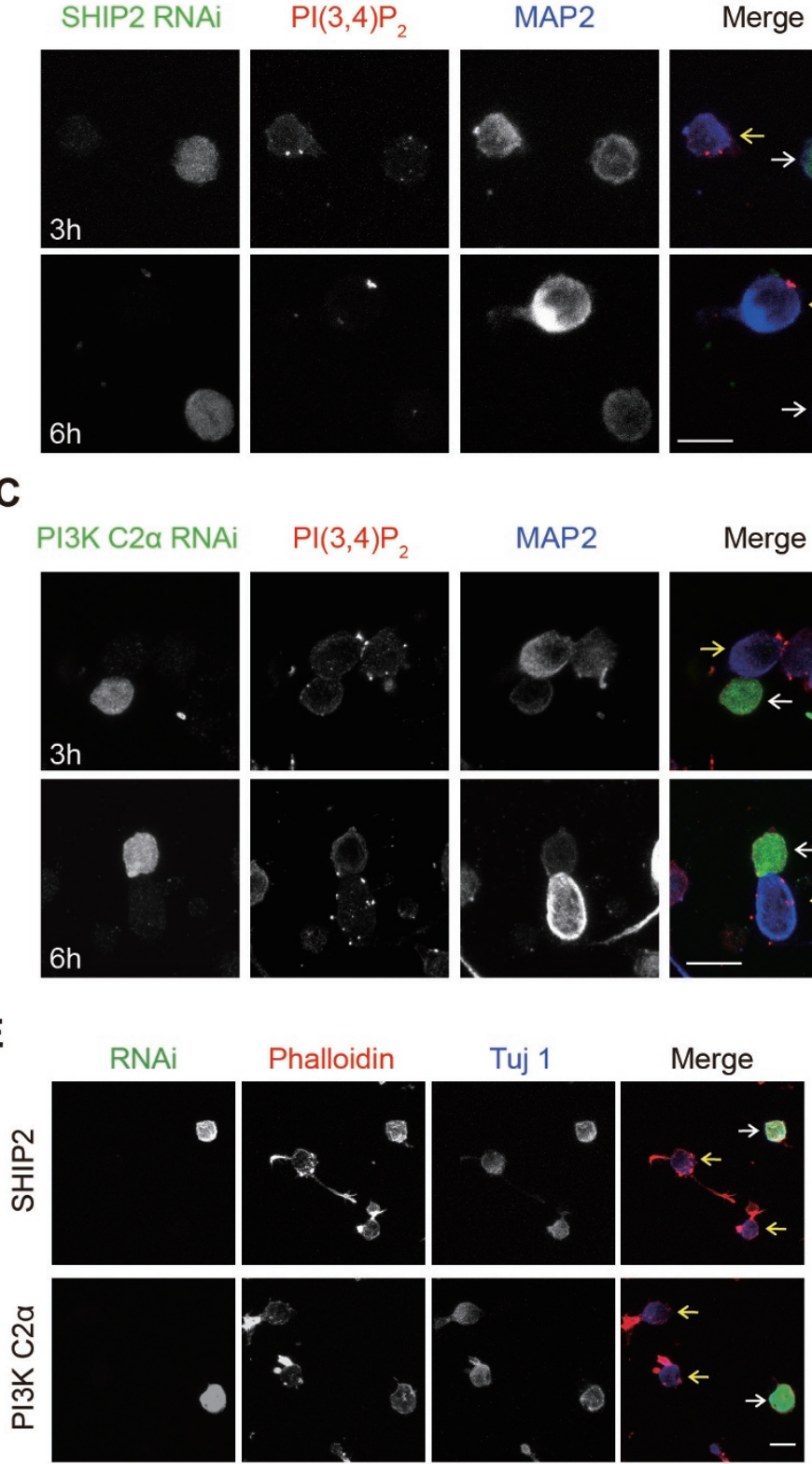

MAP2

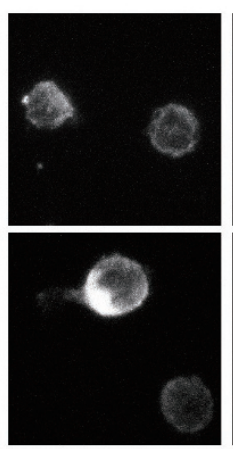

MAP2
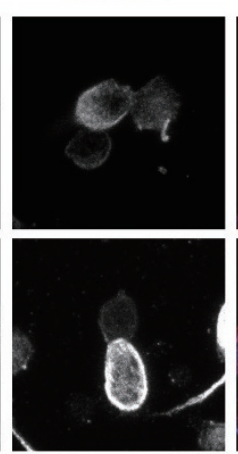

B
Merge

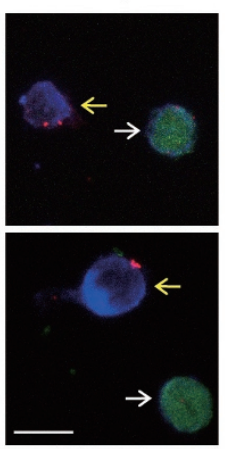

Merge
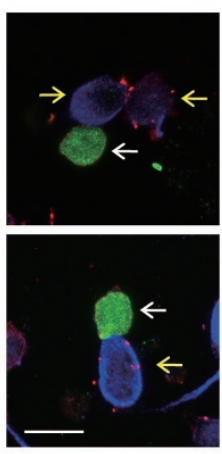

D

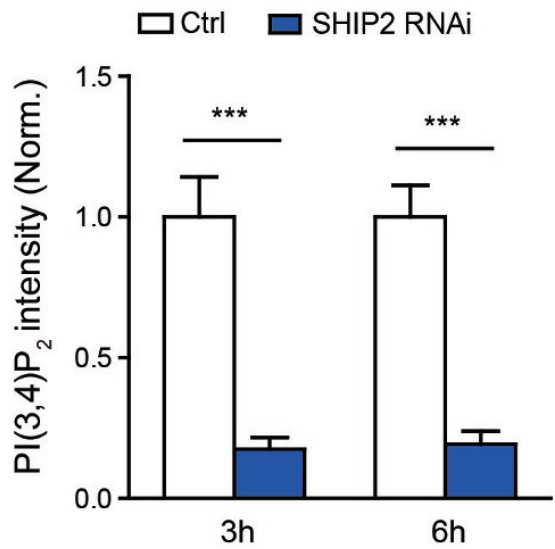

\section{$\square \mathrm{Ctrl} \square \mathrm{PI3K}$ C2 2 RNAi}

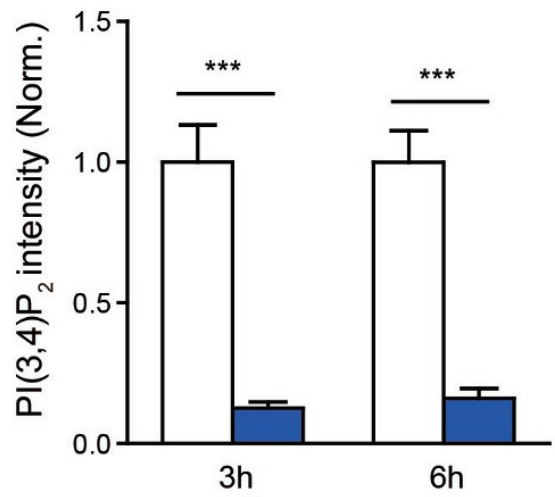

F

G
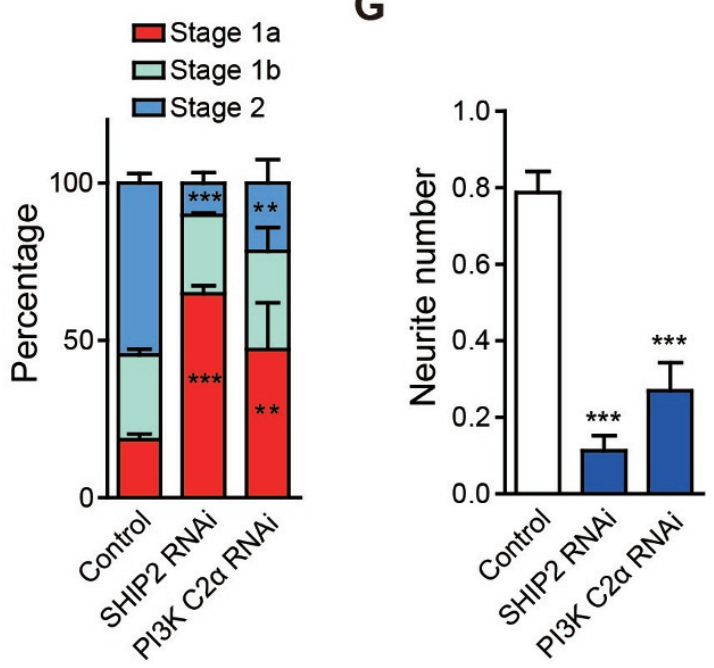

Figure $4 \mathrm{PI}(3,4) \mathrm{P}_{2}$ regulators SHIP2 and $\mathrm{PI} 3 \mathrm{~K}$ C2 $\alpha$ are required for actin aggregation and neurite initiation. (A, C) Representative images of neurons cultured from embryos in utero electroporated with constructs coexpressing GFP and SHIP2 RNAi (A) or GFP and PI3K C2 $\alpha$ RNAi (C). White arrows indicate RNAi-positive cells expressing GFP, and yellow arrows indicate nearby wild-type cells. Neurons were labeled for $\mathrm{PI}(3,4) \mathrm{P}_{2}$ (red) and MAP2 (blue). Scale bar, $10 \mu \mathrm{m}$. (B, D) Quantification of neuronal $\mathrm{PI}(3,4) \mathrm{P}_{2}$ intensity. $n=39$ and 43 neurons for Ctrl groups, 50 and 42 neurons for SHIP2 RNAi groups for each time points in B. $n=39$ and 35 neurons for Ctrl groups, 33 and 34 neurons for PI3K C2 $\alpha$ RNAi groups for each time points in $\mathbf{D}$. Unpaired $t$-tests in B and D. (E) Representative images of neurons cultured from embryos in utero electroporated with SHIP2 RNAi or PI3K C2 $\alpha$ RNAi construct. Scale bar, $10 \mu \mathrm{m}$. (F, G) Quantification of neuronal developmental stages (F) and neurite number (G). $n=235,80$ and 52 for Ctrl, SHIP2 RNAi and PI3K C2 $\alpha$ RNAi groups; two-way ANOVA followed by Bonferroni's post hoc test in $\mathbf{F}$ and one-way ANOVA followed by Dunnett's post hoc test in $\mathbf{G}$. 
A

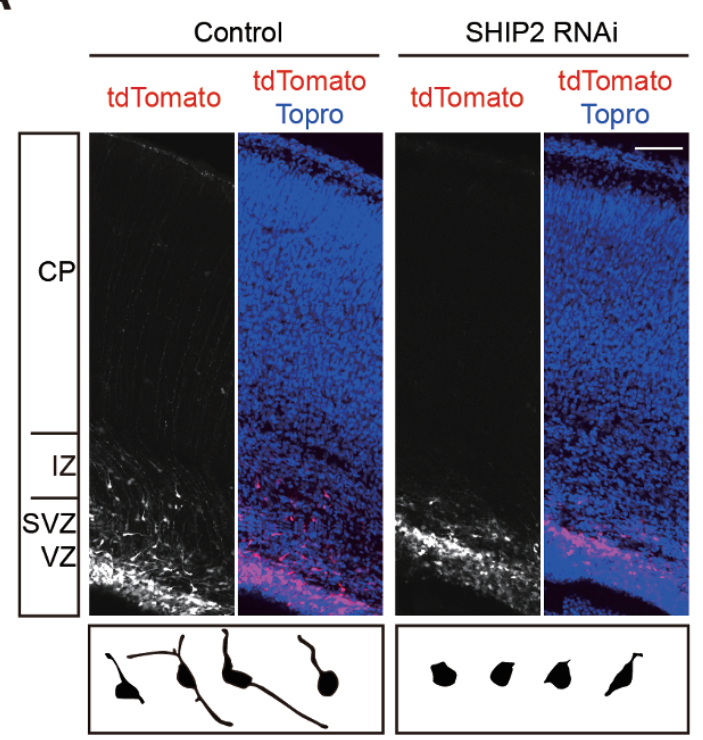

C

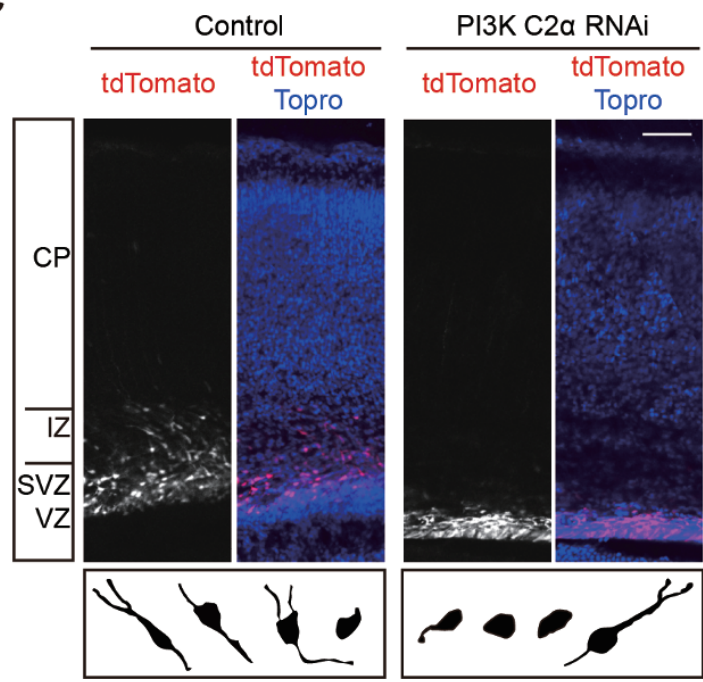

B

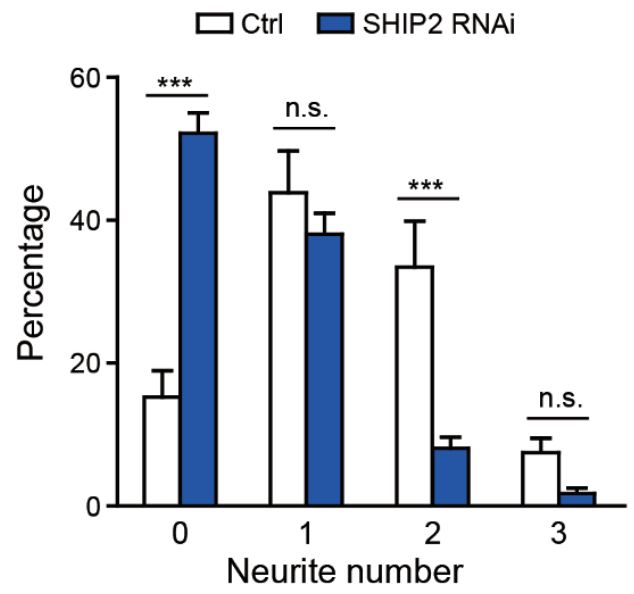

D

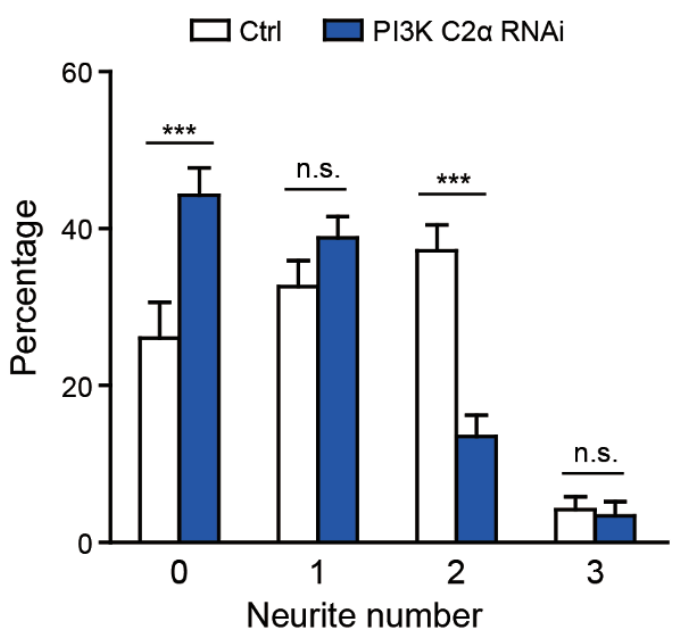

Figure 5 SHIP2 and PI3K C2 $\alpha$ are required for neurite initiation in vivo. (A, C) Representative images of rat E18.5 cortical slices from embryos in utero electroporated with constructs co-expressing tdTomato and SHIP2 RNAi (A) or tdTomato and PI3K C2 $\alpha$ RNAi (C); tdTomato was used as a morphology marker. The bottom boxes show representative neurons traced from these cortical slices. CP, cortical plate; IZ, immediately zone; (S)VZ, (sub)ventricular zone. Scale bar, $100 \mu \mathrm{m}$. (B, D) Quantification of neurite number. In B, 215 neurons from 14 frames were quantified in the Ctrl group and 423 neurons from 15 frames in the SHIP2 RNAi group; in D, 221 neurons from 10 frames were quantified in the Ctrl group and 220 neurons from 10 frames in the PI3K C2 $\alpha$ RNAi group; one-way ANOVA followed by Dunnett's post hoc test.

ure 7A-7C). The average dendrite length was increased (Figure 7D), likely as a compensatory effect of reduced primary dendrite number. Total axon length was not affected (Figure 7E). Together, these results demonstrate that $\mathrm{PI}(3,4) \mathrm{P}_{2}$, SHIP2 and PI3K C2 $\alpha$ specifically regulate neurite initiation, without affecting axon-dendrite specification and neuronal polarization.

Since SHIP2 and PI3K C2 $\alpha$ are both required for neuritogenesis and dendritogenesis, we next asked whether they were redundant. In other words, can SHIP2 overexpression compensate for reduction in PI3K C $2 \alpha$ level or vice versa. The efficiency of the RNAi-resistant overexpression constructs (SHIP2* and PI3K C2 $\alpha^{*}$ ) was first tested using western blotting (Supplementary information, Figure S3G). DIV 2 neurons were then transfected with overexpression and RNAi constructs in 
A
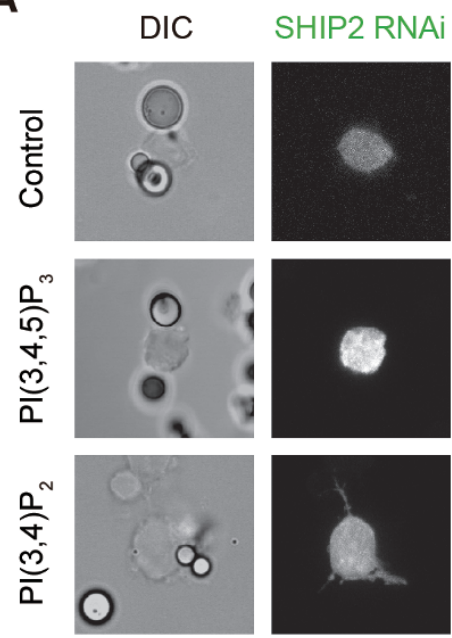

C
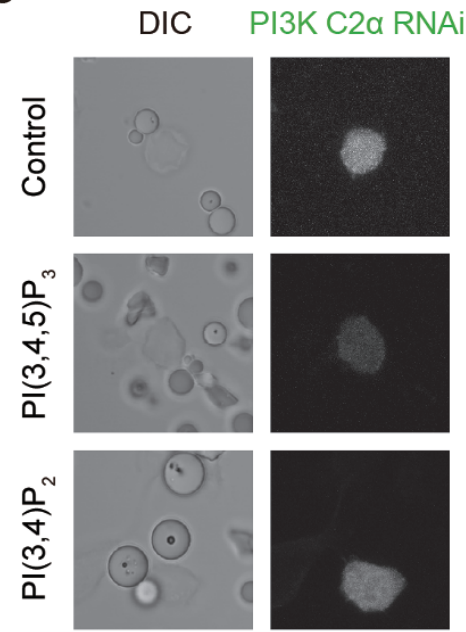
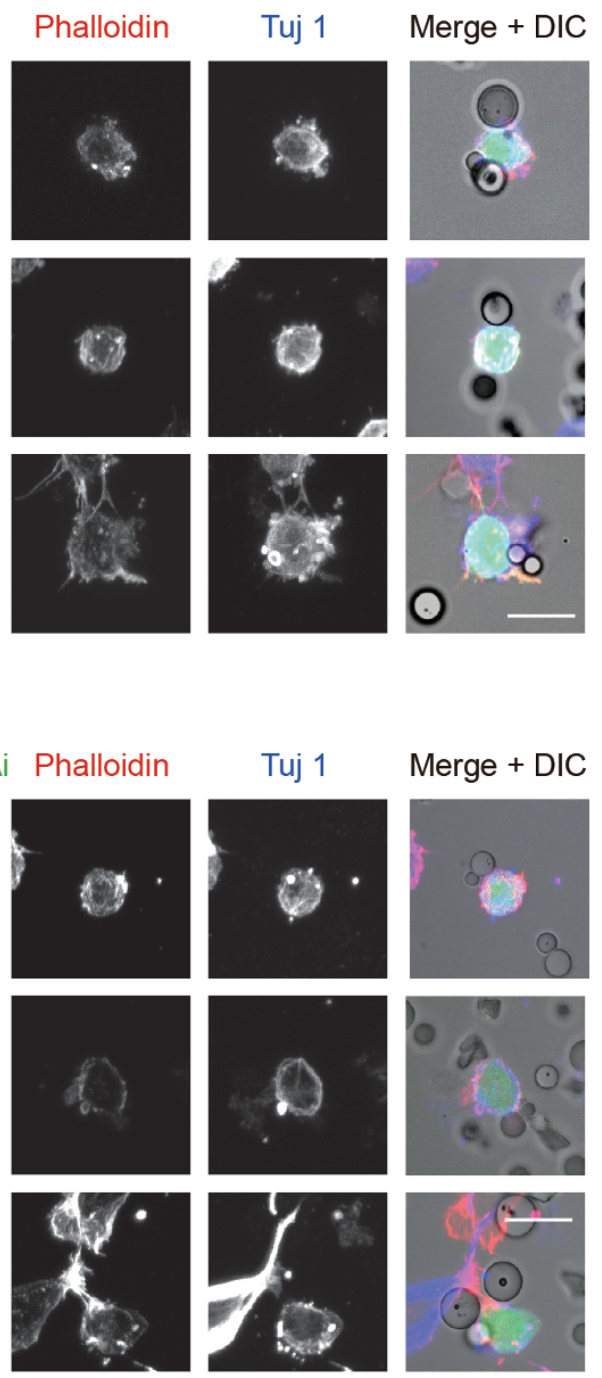

B

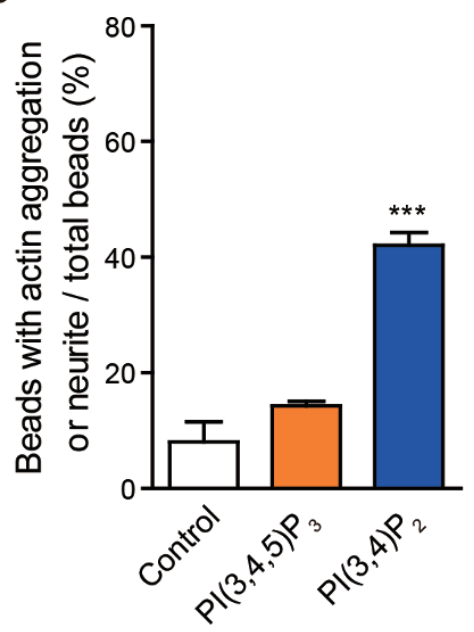

D

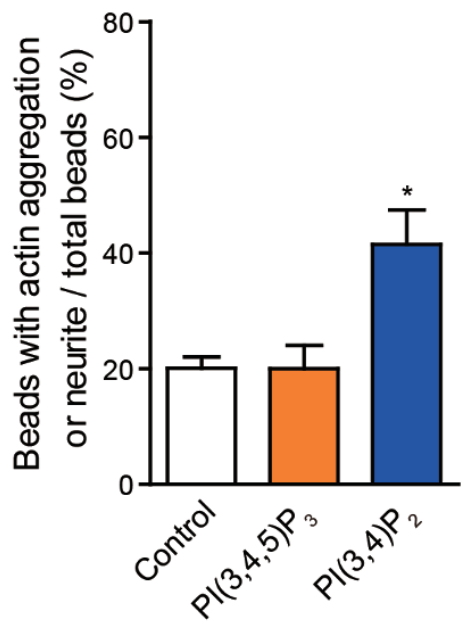

Figure $6 \mathrm{PI}(3,4) \mathrm{P}_{2}$-coated beads rescue SHIP2 RNAi and PI3K C2 $\alpha$ RNAi effects. (A, C) Representative images of neurons cultured from embryos in utero electroporated with constructs co-expressing GFP and SHIP2 RNAi (A) or GFP and PI3K C2 $\alpha$ RNAi (C), and treated with beads coated with carrier alone (control), carrier and $\mathrm{PI}(3,4,5) \mathrm{P}_{3}$ or carrier and $\mathrm{PI}(3,4) \mathrm{P}_{2}$. Neurons were labeled for phalloidin (red) and Tuj 1 (blue); beads were visualized under DIC. Scale bar, $10 \mu \mathrm{m}$. (B, D) Quantification of neuritogenesis and actin aggregation of SHIP2 RNAi neurons (B) or PI3K C2 $\alpha$ RNAi neurons (D). In B, 123 beads from 73 image frames were quantified in the control group, 125 beads from 71 frames in the $\mathrm{PI}(3,4,5) \mathrm{P}_{3}$ group and 107 beads from 73 frames in the $\mathrm{PI}(3,4) \mathrm{P}_{2}$ group. In $\mathbf{D}, 90$ beads from 59 image frames were quantified in the control group, 87 beads from 51 frames in the $\mathrm{PI}(3,4,5) \mathrm{P}_{3}$ group and 82 beads from 52 frames in the $\mathrm{PI}(3,4) \mathrm{P}_{2}$ group; one-way ANOVA followed by Dunnett's post hoc test.

different combinations, and the dendrite morphology of the resulting neurons was examined at DIV 5. Neuronal morphology was visualized by co-transfected GFP, and axons were excluded from analysis by their longer length and even diameter. The results showed that SHIP2 bidirectionally regulated the number of primary dendrites: its overexpression significantly increased dendrite number, while its RNAi significantly reduced dendrite number
(Figure 7F and 7G). Overexpression of SHIP2* completely rescued the reduction in primary dendrite number induced by its RNAi (Figure 7F and 7G), demonstrating specificity of the RNAi. Similar results were observed for PI3K C2 $\alpha$ (Figure 7F and 7G). Interestingly, when PI3K $\mathrm{C} 2 \alpha^{*}$ was used to rescue SHIP2 RNAi, or SHIP2* was used to rescue PI3K C2 $\alpha$ RNAi, primary dendrite numbers were not significantly different from SHIP2 RNAi 
A

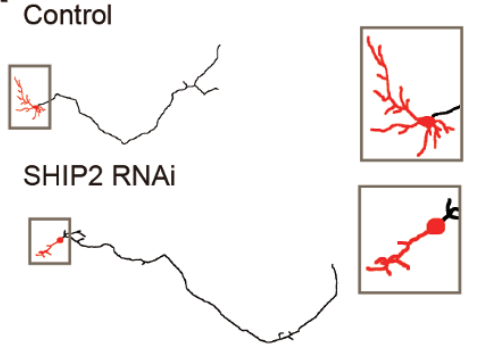

PI3K C2a RNAi

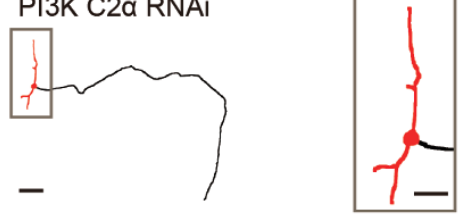

F

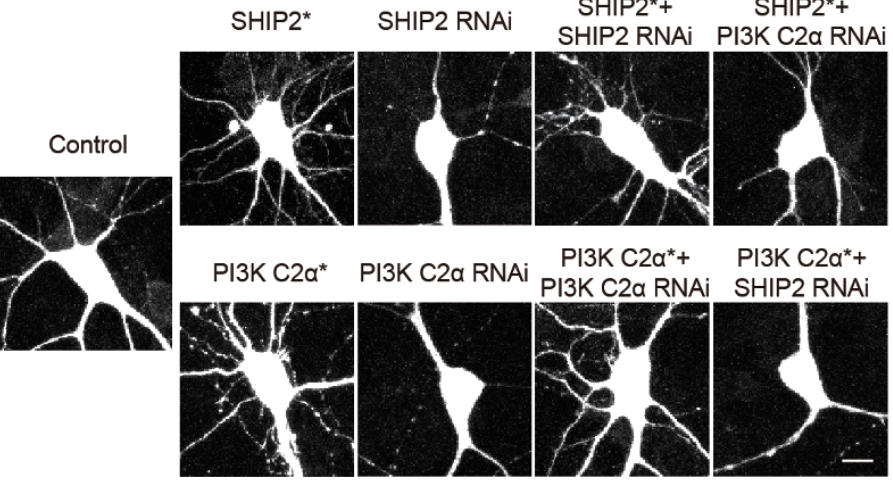

H

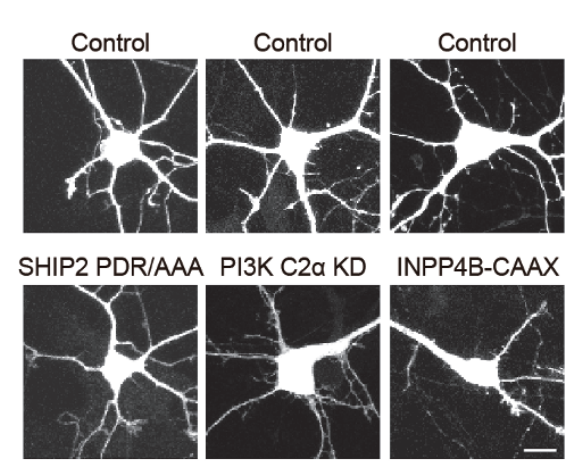

B

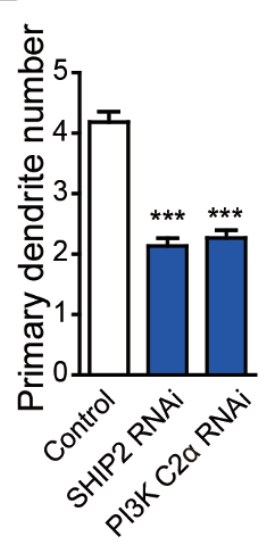

I

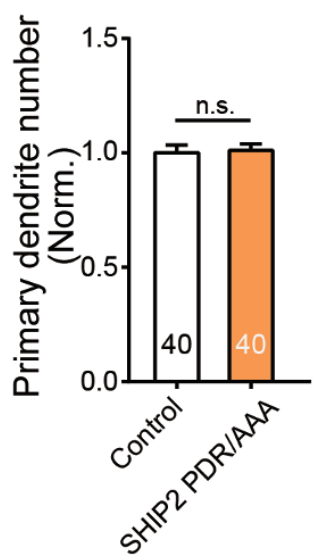

C

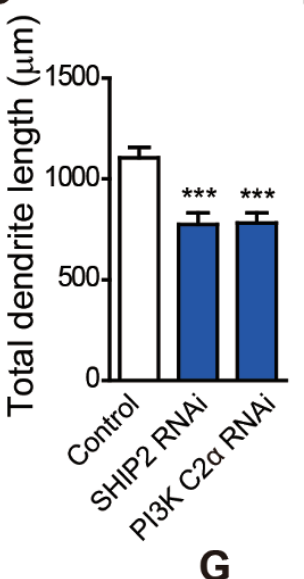

D E

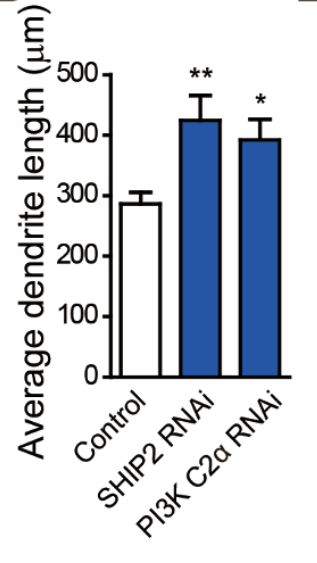

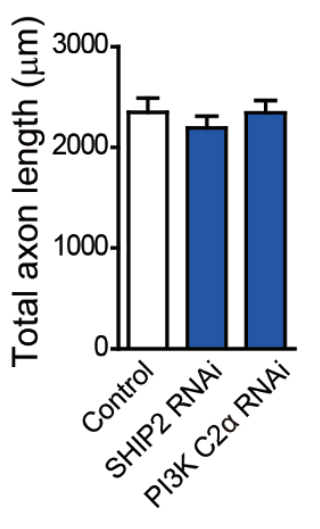

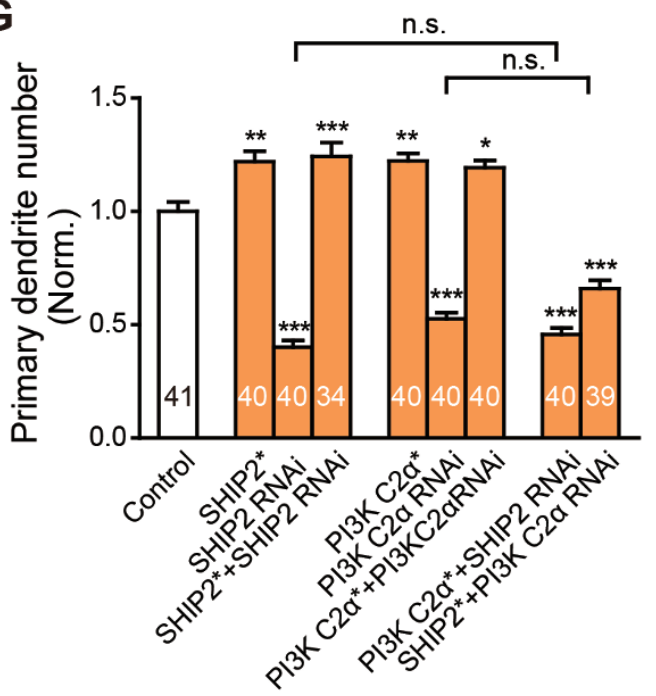

$\mathrm{K}$

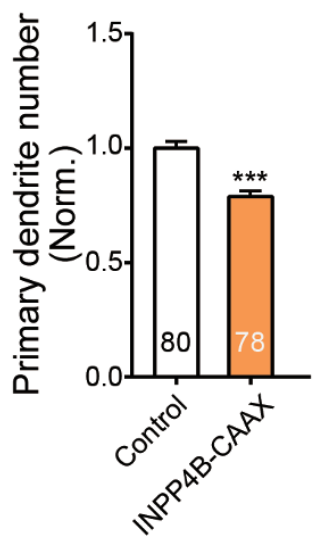

Figure 7 SHIP2 and PI3K C2 $\alpha$ are specifically required for dendritogenesis and are non-redundant in their functions. (A) Examples of neuronal tracings of DIV 3 neurons, dendrites shown in red, axons in black. Scale bar, $200 \mu \mathrm{m}$, or $100 \mu \mathrm{m}$ for the boxed insets. (B-E) Quantification of primary dendrite number (B), total dendrite length (C), average dendrite length (D) and total axon length (E). $n=44,46$ and 45, respectively, for the three groups in each graph; one-way ANOVA followed by Dunnett's post hoc test. (F, H) Representative images of DIV5 neurons, conditions as indicated. Neurons were co-transfected with GFP to visualize their morphology. Scale bar, $10 \mu \mathrm{m}$. (G) Quantification of primary dendrite number of neurons shown in $\mathbf{F}$. $n$ as indicated inside bar graphs; one-way ANOVA followed by Tukey's post hoc test. (I-K) Quantification of primary dendrite number of neurons shown in H. $n$ as indicated inside bar graphs; unpaired $t$-tests. 
or PI3K C $2 \alpha$ RNAi conditions, respectively (Figure 7F and $7 \mathrm{G})$, demonstrating that the two $\mathrm{PI}(3,4) \mathrm{P}_{2}$-generating pathways are non-redundant, and that both are required for normal neuronal morphogenesis.

To investigate whether the enzymatic activities of SHIP2 and PI3K C2 $\alpha$ are required for the observed effects, we overexpressed 5'-phosphatase-defective SHIP2 (SHIP2 PDR/AAA) $[58,59]$ and kinase-inactive PI3K C2 $\alpha$ (PI3K C2 $\alpha$ KD) $[57,60]$. The results showed that neither manipulation significantly affected primary dendrite number (Figure 7H-7J), indicating the requirement of the enzymatic functions of these proteins in regulating primary dendrite number. In complementary experiments, we overexpressed the $\mathrm{PI}(3,4) \mathrm{P}_{2}$-specific 4-phosphatase, inositol-3,4-bisphosphate 4-phosphatase type II (INPP4B), and targeted it to the membrane using a carboxy-terminal CAAX-box prenylation sequence (INPP4B-CAAX) [57]. INPP4B-CAAX overexpression significantly reduced primary dendrite number (Figure $7 \mathrm{H}$ and $7 \mathrm{~K}$ ), similar to the effects of SHIP2 RNAi or PI3K C $2 \alpha$ RNAi, providing further evidence for the requirement of $\mathrm{PI}(3,4) \mathrm{P}_{2}$ in regulating primary dendrite number.

$N$-WASP is necessary and sufficient for mediating actin aggregation and neurite initiation downstream of $P I(3,4)$ $\mathrm{P}_{2}$

Having demonstrated a critical role of $\mathrm{PI}(3,4) \mathrm{P}_{2}$ and its upstream enzymes SHIP2 and PI3K C2 $\alpha$ in regulating actin aggregation and neurite initiation, we next asked what molecules mediated the downstream actin remodeling process. A promising candidate is the actin-remodeling protein lamellipodin, which binds to $\mathrm{PI}(3,4) \mathrm{P}_{2}$ and recruits Ena/VASP, thereby promoting the assembly of actin filaments at the plasma membrane [61, 62]. Unfortunately, no significant effects on primary dendrite number were observed in experiments using three independent RNAi constructs previously reported to knock down lamellipodin [56, 62, 63] (Supplementary information, Figure S4A and S4B). Instead, we observed significant reduction in total dendrite branch tip number (TDBTN), a measure of dendrite complexity (Supplementary information, Figure S4C and S4D). Together, these results suggest that lamellipodin contributes to dendrite morphogenesis, but does not regulate primary dendrite number. We then screened through other molecules previously reported to bind to $\mathrm{PI}(3,4) \mathrm{P}_{2}$ and capable of polymerizing actin [57, 64-66] and identified neural Wiskott-Aldrich syndrome protein (N-WASP) as a potential downstream target. N-WASP is a member of Wiskott-Aldrich family of proteins, originally known for its high expression in neural tissue [67-69]. Upon upstream signaling, N-WASP stimulates local actin polymerization by recruiting the actin-related protein 2/3 (Arp2/3) complex [70-72]. To confirm the interaction between $\mathrm{PI}(3,4) \mathrm{P}_{2}$ and N-WASP, we performed co-immunoprecipitation using PI $(3,4)$ $\mathrm{P}_{2}$-coated beads, and showed that they specifically bound to GST-N-WASP B motif, a domain in N-WASP previously shown to bind to PIs [66] (Figure 8A; please also see the Discussion section). In complementary experiments, we used Stinger, a fluorescence resonance energy transfer (FRET)-based probe of active N-WASP, whose FRET signaling is reduced upon N-WASP activation and opening of its inactive, closed conformation [73], to assay the ability of $\mathrm{PI}(3,4) \mathrm{P}_{2}$ to activate N-WASP. The results showed that $\mathrm{PI}(3,4) \mathrm{P}_{2}$ application significantly reduced FRET signaling of the Stinger probe, demonstrating its ability to activate N-WASP (Figure $8 \mathrm{~B}$ and $8 \mathrm{C}$ ).

In terms of the requirement of N-WASP for neuronal morphogenesis, when cultured neurons were treated with the N-WASP chemical inhibitor wiskostatin [74] $(1 \mu \mathrm{g} /$ $\mathrm{ml}$ ) for $6 \mathrm{~h}, 74.2 \% \pm 0.6 \%$ of neurons were stalled at stage $1 \mathrm{a}$, as compared to only $13.5 \% \pm 5.4 \%$ in control cultures (Figure $8 \mathrm{D}$ and $8 \mathrm{E}$ ). In addition to inhibiting neuronal development, wiskostatin treatment also significantly blocked neurite initiation (Figure 8F). To further examine the effect of N-WASP activity on neurite initiation, we generated a constructively active (N-WASP CA) form of the protein by overexpressing a membrane-anchored form of its C-terminal verprolin homology, cofilin homology and acidic (VCA) domain. The VCA domain of N-WASP and related proteins has been shown to coordinate binding between actin monomers and the Arp2/3 complex $[68,69]$. Overexpression of N-WASP CA indeed significantly increased primary dendrite number, as compared to control neurons expressing GFP only, or those expressing the CD8 membrane anchor (Figure $8 \mathrm{G}$ and $8 \mathrm{H}$ ). Since under resting conditions, the N-terminal region of N-WASP binds to the VCA region and prevents its activation $[68,69]$, full-length N-WASP, even when overexpressed, is still subject to endogenous regulation and had no significant effects (Figure 8G and 8H). In contrast, overexpression of the $\mathrm{N}$-terminal region of N-WASP (N-WASP DN), which binds to exposed VCA domains and sequesters them from action, significantly reduced primary dendrite number (Figure $8 \mathrm{G}$ and $8 \mathrm{H}$ ). Together, these results demonstrate a requirement for N-WASP during dendritogenesis.

We next tested whether N-WASP functioned downstream of SHIP2 and PI3K C2 $\alpha$ using two complementary sets of epistasis experiments. Our results showed that coexpression of N-WASP CA with SHIP2 RNAi or PI3K C2 $\alpha$ RNAi construct resulted in a phenotype indistinguishable from overexpression of N-WASP CA alone 
A

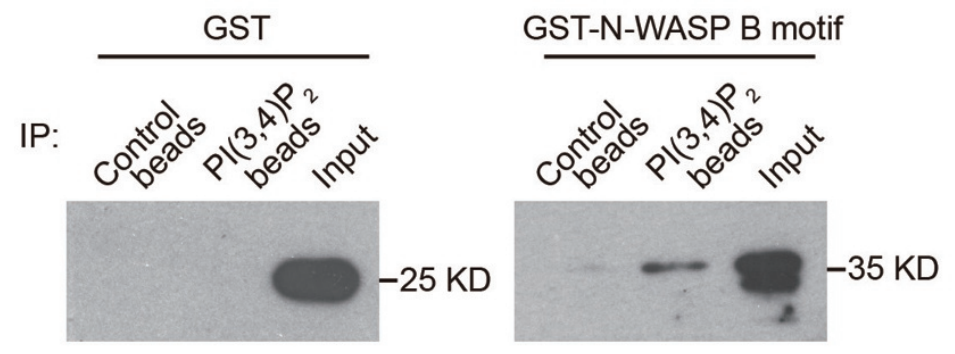

IB: GST

D

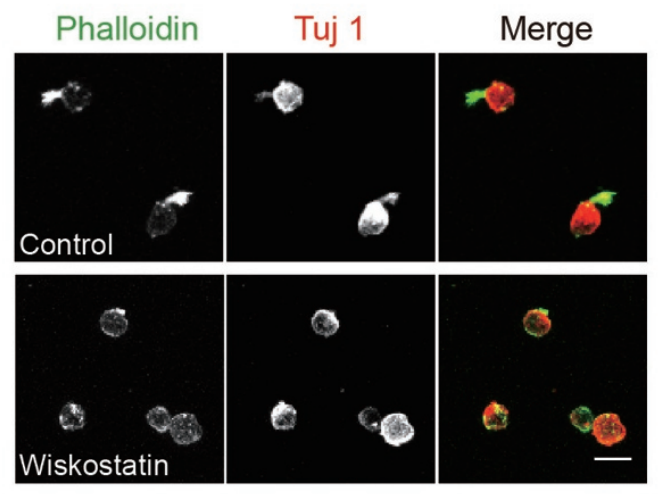

G

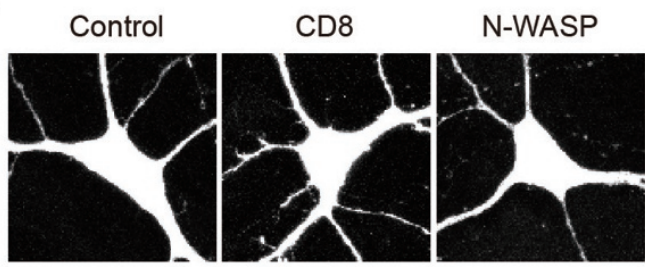

N-WASP CA N-WASP DN

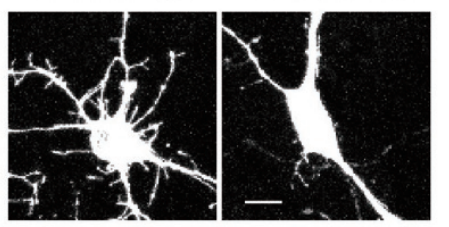

H
B

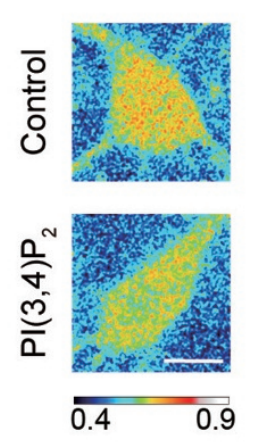

E

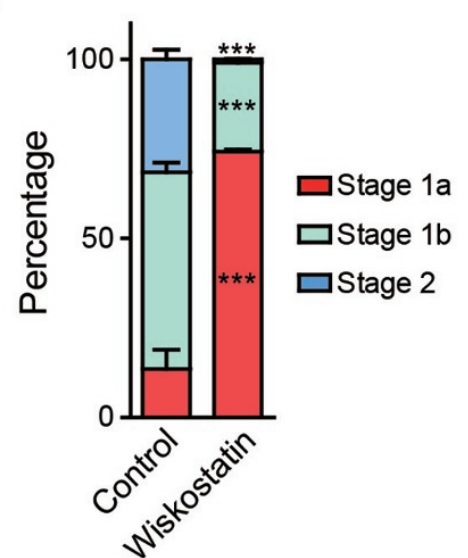

C

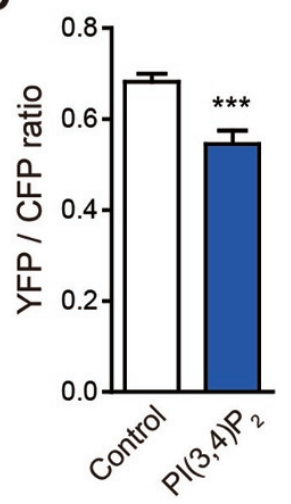

F

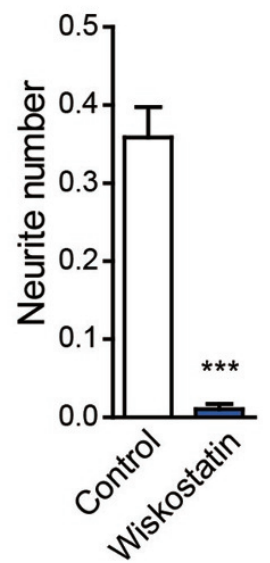

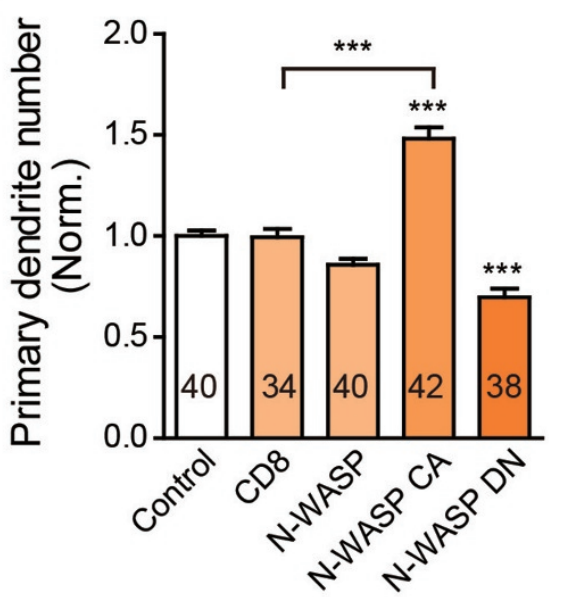

Figure 8 N-WASP regulates actin aggregation and dendritogenesis downstream of $\mathrm{PI}(3,4) \mathrm{P}_{2}$. (A) Immunoblot using anti-GST antibody showing that N-WASP B motif specifically interacted with $\mathrm{PI}(3,4) \mathrm{P}_{2}$. (B) Representative images of neurons expressing Stinger, a FRET-based N-WASP activity probe; neurons were treated with carrier alone (control) or carrier and $\mathrm{PI}(3,4)$ $\mathrm{P}_{2}$. Images are shown using Royal look-up table from Fiji/ImageJ. Scale bar, $10 \mu \mathrm{m}$. (C) Quantification of neuronal YFP/CFP ratio. $n=20$ neurons for each group; unpaired $t$-test. (D) Representative images of neurons treated with DMSO vehicle (Control) or $1 \mu \mathrm{g} / \mathrm{ml}$ wiskostatin. Scale bar, $10 \mu \mathrm{m}$. (E, F) Quantification of neuronal developmental stages (E) and neurite number (F). $n=201$ and 198 neurons for control and wiskostatin groups, respectively; in E, two-way ANOVA followed by Bonferroni's post hoc test was used; in F, unpaired t-test was used. (G) Representative images of DIV5 neurons, conditions as indicated. Neurons were co-transfected with GFP to visualize their morphology. Scale bar, $10 \mu \mathrm{m}$. (H) Quantification of primary dendrite number of neurons shown in G. $n$ as indicated inside bar graphs, one-way ANOVA followed by Tukey's post hoc test. 
(Figure 9A and 9B). In the complementary set of experiments, overexpression of N-WASP DN together with SHIP2 or PI3K C $2 \alpha$ mimicked the effect of N-WASP DN overexpression alone (Figure 9C and 9D). Togeth$\mathrm{er}$, these results demonstrate that N-WASP functioned downstream of SHIP2 and PI3K C2 $\alpha$ to regulate neuronal morphogenesis. We note that when N-WASP CA was overexpressed together with SHIP2 RNAi or PI3K C2 $\alpha$ RNAi construct, neurons looked relatively unhealthy, suggesting a negative impact on neuronal development from conflicting neuritogenesis signals.

Since N-WASP mediated actin aggregation by recruiting Arp $2 / 3$ complex, we next tested whether components of Arp2/3 complex functioned downstream of N-WASP during neuritogenesis. As shown in Figure 9E and quantitated in Figure 9F, Arp3 RNAi completely blocked the effect of N-WASP CA, whereas Arp3 overexpression fully rescued the effect of N-WASP DN. Together, these results demonstrate that N-WASP mediated the effect of $\mathrm{PI}(3,4) \mathrm{P}_{2}$ signaling on actin aggregation and neuritogenesis via the Arp2/3 complex.

\section{Discussion}

In summary, we demonstrated that SHIP2- and PI3K $\mathrm{C} 2 \alpha$-mediated local clustering of the phosphoinositide $\mathrm{PI}(3,4) \mathrm{P}_{2}$ serves as a signal for regulating actin aggregation and neuritogenesis, a process mediated downstream by N-WASP- and Arp2/3-dependent actin polymerization (Figure 10). Our results demonstrate a novel signaling role for $\mathrm{PI}(3,4) \mathrm{P}_{2}$ in early neuronal development and identify a membrane-initiated signaling cascade for regulating neuritogenesis.

\section{Regulation of actin aggregation by $\mathrm{PI}(3,4) \mathrm{P}_{2}$}

The identification of $\mathrm{PI}(3,4) \mathrm{P}_{2}$ as the upstream signal for regulating actin aggregation during neuritogenesis is very surprising, as it was generally considered to be a minority PI in the plasma membrane with few known signaling functions [29-34]. By comparison, the related $\mathrm{PI}(4,5) \mathrm{P}_{2}$ can be hydrolyzed into the important secondary messengers $\mathrm{IP}_{3}$ and DAG to activate downstream signaling cascades [75], and has also been extensively studied for its regulation of actin polymerization [29-32]. Another member of the same family, PIP $_{3}$, has been shown to regulate other early neuronal developmental processes, including axon specification and neuronal polarization [35-37], and to activate the protein kinases PDK and AKT/PKB pathways and their downstream signaling [76]. However, previous studies have not examined the subcellular localization of these PIs in very young neurons. The striking punctate subcellular localization of $\mathrm{PI}(3,4)$
$\mathrm{P}_{2}$ and the co-localization of these puncta with F-actin aggregates (Figure 1), in contrast to the relatively diffuse localization of $\mathrm{PI}(4,5) \mathrm{P}_{2}$ or $\mathrm{PIP}_{3}$ at the same developmental stage, provide convincing evidence that $\mathrm{PI}(3,4) \mathrm{P}_{2}$ is a key regulator of actin aggregate formation, a result confirmed by our subsequent pharmacological and molecular manipulations (Figures 2 and 3).

Why use a minority PI with few signaling roles to regulate actin aggregation during neurite initiation? We believe that there may be distinct advantages. Since most neurons initially only have one or two neurites extending from the cell body [28], and the formation of each requires assembly of a large actin aggregate [22, 23], neuritogenesis is essentially an all-or-none process. Previous modeling and experimental work have shown that spatially directed synthesis and degradation of PIs can result in self-organizing cell polarization [77]. Interestingly, positive feedback alone only generates polarization when the concentration of the polarity marker is limiting [77]. The low concentrations of $\mathrm{PI}(3,4) \mathrm{P}_{2}$ in the cell fulfills this criterion. Consistent with a positive feedback mechanism during neurite initiation, we found that inhibition of actin dynamics with jasplakinolide for the prolonged duration of $6 \mathrm{~h}$ reduced $\mathrm{PI}(3,4) \mathrm{P}_{2}$ puncta size (Figure 3 ). Another advantage of $\mathrm{PI}(3,4) \mathrm{P}_{2}$-dependent signaling is that it has been shown to be capable of sustained signaling, a characteristic favorable to the slow, but relatively irreversible, process of neuritogenesis [78]. In non-neuronal cells, $\mathrm{PI}(3,4) \mathrm{P}_{2}$ has also been shown to signal the formation of podosome/invadopodia, F-actin-rich structures with similarities to neurites, between the plasma membrane and neighboring extracellular matrix [79]. Thus, the ability of $\mathrm{PI}(3,4) \mathrm{P}_{2}$ to direct growth of a filopodial extension from the cell body under specific circumstances may be a general cellular feature.

Having identified the molecular mechanism underlying neurite initiation, it is interesting to retrospectively discuss why related PIs, namely, $\mathrm{PI}(3,4) \mathrm{P}_{2}$ and $\mathrm{PIP}_{3}$, regulate neurite initiation and neuronal polarization, respectively $[35,36]$. Even though neuritogenesis is defined as step 2 of neuronal development and neuronal polarization as step $3[1,2]$, they in practice occur within a similar time window, with the third and fourth neurites extending, while the initial two neurites establish polarity. Thus, different signaling cascades need to regulate these distinct cellular events. In fact, while $\mathrm{PI}(3,4) \mathrm{P}_{2}$ puncta were observed at the cell body, $\mathrm{PIP}_{3}$ enrichment was only observed at the tip of growth cones [35, 36], suggesting that $\mathrm{PI}(3,4) \mathrm{P}_{2}$ determines the sites of neurite initiation, whereas $\mathrm{PIP}_{3}$ regulates their differentiation and growth. Since PI content in the membrane is limited, having different PIs regulate each of these processes avoids con- 
A

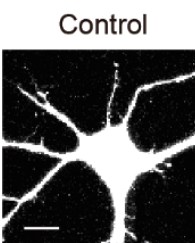

N-WASP CA

SHIP2 RNAi PI3K C2 $\alpha$ RNAi

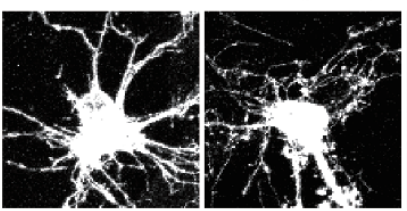

C

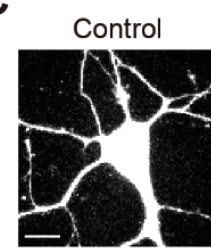

N-WASP DN

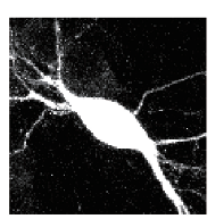

SHIP2 + $\mathrm{N}-$ WASP DN

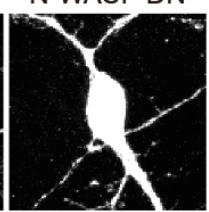

$\mathrm{PI} 3 \mathrm{~K} \mathrm{C2a+}$ N-WASP DN

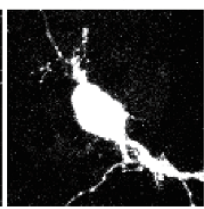

B

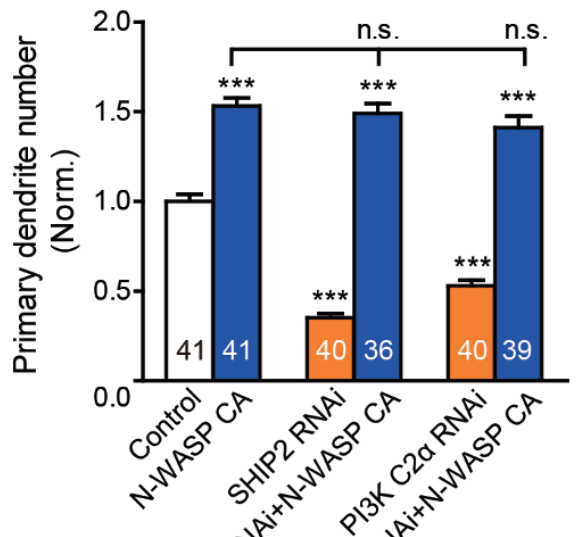

D

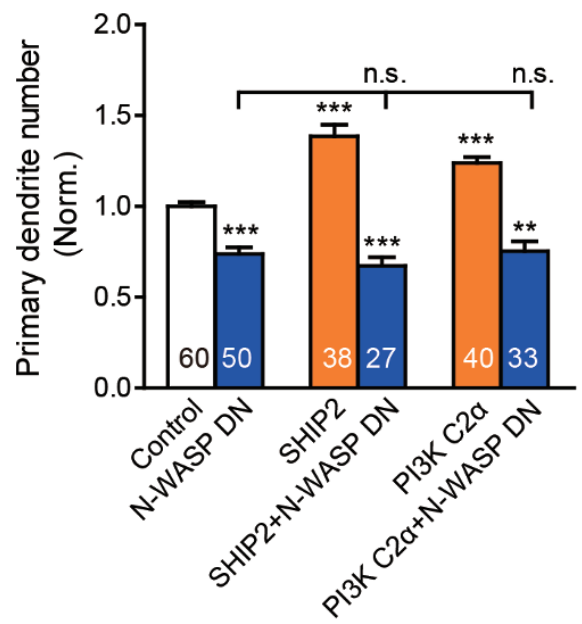

$\mathbf{F}$

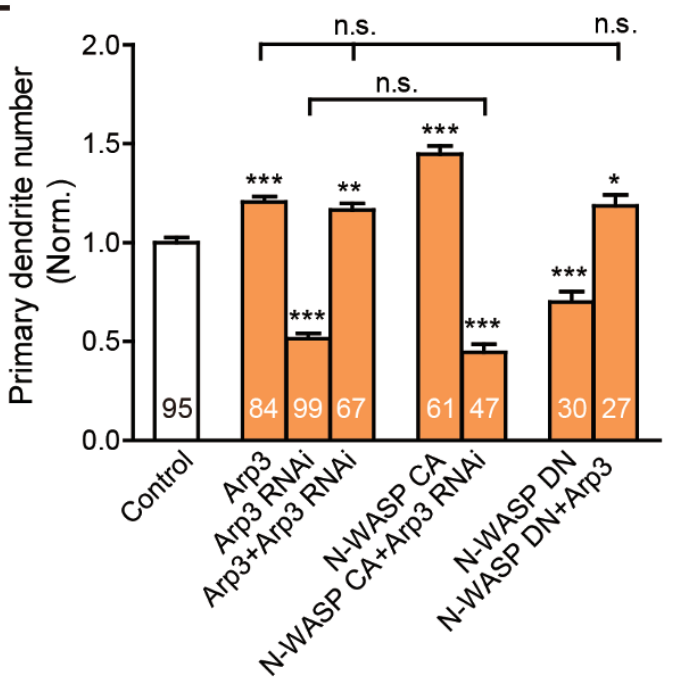

Figure 9 N-WASP/Arp complex mediates $\mathrm{PI}(3,4) \mathrm{P}_{2}$-dependent dendritogenesis. (A, C, E) Representative images of DIV 5 neurons, conditions as indicated. Neurons were co-transfected with GFP to visualize their morphology. Scale bar, $10 \mu \mathrm{m}$. (B, D, F) Quantification of primary dendrite number in A, C, E, respectively. $n$ as indicated inside bar graphs; one-way ANOVA followed by Tukey's post hoc test. 


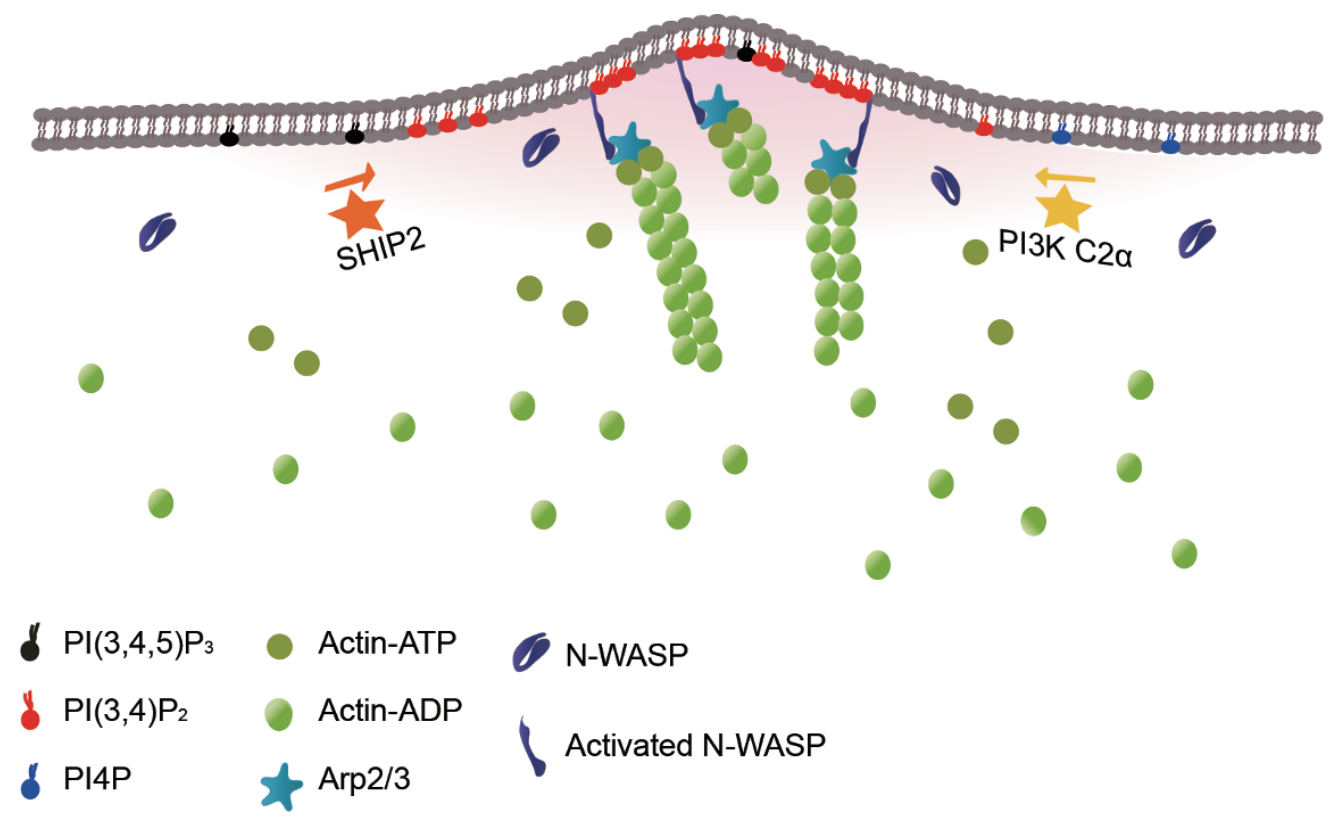

Figure $10 \mathrm{~A}$ working model of the mechanism underlying $\mathrm{PI}(3,4) \mathrm{P}_{2}$-dependent actin aggregation and neurite initiation.

current occurrence of both processes. Regulation of both of these important developmental processes by PIs also speaks to the critical role of PI signaling in cellular functioning.

Non-redundant regulation of $\mathrm{PI}(3,4) \mathrm{P}_{2}$ level by SHIP2 and PI3K C2 $\alpha$

We demonstrated that SHIP2 and PI3K C2 $\alpha$ contributed non-redundantly and significantly to regulating $\mathrm{PI}(3,4) \mathrm{P}_{2}$ level during neuritogenesis and dendritogenesis (Figures 4,5 and 7). These enzymes generate $\mathrm{PI}(3,4) \mathrm{P}_{2}$ via different mechanisms: SHIP2 dephosphorylates $\mathrm{PIP}_{3}$, whereas PI3K C2 $\alpha$ phosphorylates PI(4)P [29-32, 34, $50,53]$. The requirement of two distinct mechanisms to generate one molecule for a specific signaling event is intriguing. We propose that this is necessary, because a high concentration of $\mathrm{PI}(3,4) \mathrm{P}_{2}$ is required at the site of neurite initiation within a short time window to aggregate actin. Thus, all available PI sources at the site need to be recruited. Previous studies showed that the most abundant PIs in the membrane at resting state are $\mathrm{PI}(4,5)$ $\mathrm{P}_{2}$ and PI(4)P [29-32]. PI3K C2 $\alpha$ generates $\mathrm{PI}(3,4) \mathrm{P}_{2}$ from PI(4)P. To be used, available PI $(4,5) \mathrm{P}_{2}$ first needs to be converted to $\mathrm{PIP}_{3}$. We showed in Figure 2 that interfering with $\mathrm{PIP}_{3}$ generation from $\mathrm{PI}(4,5) \mathrm{P}_{2}$ using LY294002 had a small but significant effect on reducing neurite initiation, albeit less markedly than inhibiting SHIP2 with AS1949490. Thus, although conversion of $\mathrm{PI}(4,5) \mathrm{P}_{2}$ to $\mathrm{PIP}_{3}$ is not rate limiting in generating $\mathrm{PI}(3,4)$
$\mathrm{P}_{2}$, it contributes significantly. Through concerted effort, SHIP2 and PI3K C2 $\alpha$ generate a sufficiently high level of $\mathrm{PI}(3,4) \mathrm{P}_{2}$ to aggregate actin and determine the site of neurite initiation.

$N$-WASP as a downstream mediator of PI(3,4) $P_{2}$ signaling during neuritogenesis

Having determined how $\operatorname{PI}(3,4) \mathrm{P}_{2}$ is generated, we next addressed the mechanism by which it clusters actin. Proteins including lamellipodin, N-WASP, profilin, sorting nexin-9 (SNX9) and tandem PH-domain-containing proteins, TAPP1 and TAPP2, have been previously shown to bind to $\mathrm{PI}(3,4) \mathrm{P}_{2}[56,57,62,64-66]$. Lamellipodin was an attractive candidate because it binds specifically to $\mathrm{PI}(3,4) \mathrm{P}_{2}$ [62]. Unfortunately, lamellipodin RNAi reduced dendrite complexity without affecting primary dendrite number (Supplementary information, Figure S4). We then turned to N-WASP, because it has been reported to bind to PIs cooperatively [66]. Specifically, the polybasic motif in the $\mathrm{N}$-terminal autoinhibition domain of N-WASP has been shown to bind to PI $(4,5)$ $\mathrm{P}_{2}$, as well as $\mathrm{PI}(3) \mathrm{P}, \mathrm{PI}(3,4) \mathrm{P}_{2}$ and $\mathrm{PIP}_{3}$, in a multivalent manner. This raises an issue of specificity, as $\mathrm{PI}(4,5)$ $\mathrm{P}_{2}$ is abundant in neurons and also binds to N-WASP. How might N-WASP specifically interact with $\mathrm{PI}(3,4) \mathrm{P}_{2}$ during neurite initiation? We believe that the answer lies in the differential subcellular localization of these PIs in early developing neurons. As shown in the structured illumination microscopy (SIM) images in Supplementary 
information, Figure $\mathrm{S} 5, \mathrm{PI}(4,5) \mathrm{P}_{2}$ mostly localized to the nucleus $6 \mathrm{~h}$ after plating. Thus, $\mathrm{PI}(4,5) \mathrm{P}_{2}$ is not in the same subcellular compartment as cytoplasmic N-WASP to regulate its activity.

The ability to bind to PIs cooperatively means that $\mathrm{N}$-WASP responds to the changes in PIs in a switchlike fashion, with the transition from the fully repressed state to the fully activated state occurring above a sharp threshold [66]. This characteristic is highly desirable for regulating the all-or-none process of neurite initiation. In addition to binding PIs cooperatively, N-WASP also binds to the GTP-bound form of Cdc42 through its GTPase binding domain (GBD) [71, 72]. Consistent with the physical proximity between the polybasic motif and GBD, costimulation with Cdc42 lowers the activation threshold of PIs [66]. Furthermore, Cdc42 itself has also been shown to positively regulate dendritogenesis: expression of its constitutively active form increased primary dendrite number, while expression of the dominant negative form reduced them [80]. Given the established role that $\mathrm{Cdc} 42$ and other small Rho GTPases in regulating neuronal morphogenesis and actin dynamics [81-83], the cooperativity between $\mathrm{PI}(3,4) \mathrm{P}_{2}$ and $\mathrm{Cdc} 42$ adds an additional layer of regulation to neuritogenesis.

\section{Regulation of initial $\mathrm{PI}(3,4) \mathrm{P}_{2}$ clustering}

Even though we initially studied PIs because they are membrane-bound signals that do not require extracellular signaling and thus likely function as intrinsic regulators of neuritogenesis, it is still interesting to speculate how $\mathrm{PI}(3,4) \mathrm{P}_{2}$ clusters are initially generated. We surmise that small $\mathrm{PI}(3,4) \mathrm{P}_{2}$ clusters are initially generated by stochastic mechanisms, and then these clusters are amplified through positive feedback mechanisms for $\mathrm{PI}(3,4) \mathrm{P}_{2}$ clustering and N-WASP/Arp-dependent actin aggregation. While the initial concentration and localization of different types of PIs within the membrane likely contribute to where initial clustering likely occurs, the subcellular localization of upstream enzymes SHIP2 and PI3K C $2 \alpha$ are probably not major determinants, as suggested by their relatively diffuse immunostaining (Supplementary information, Figure S3C-S3F). As to why this might be the case, we propose the following likely explanations: (1) it is the activity, rather than the subcellular localization of these enzymes, that are most critical; (2) additional, yet unidentified factors, contribute to this process; and (3) a stochastic increase in local $\mathrm{PI}(3,4) \mathrm{P}_{2}$ level at one of the many sites containing SHIP2 and/or PI3K C2 $\alpha$ activates a positive feedback process that then amplifies the signal. We particularly favor the third possibility of a stochastically initiated positive feedback process, as it is mechanistically highly flexible and adaptable. We focused on the likelihood of phospholipids as upstream regulators of local actin aggregation for this very reason. In vivo, normal brain development and organization can occur in the absence of synaptic transmission [84], suggesting critical contribution of intrinsic mechanisms to early neuronal development processes, including neurite initiation.

\section{Materials and Methods}

All animal procedures were approved by the Institutional Animal Care and Use Committee of the Institute of Neuroscience, Chinese Academy of Science (Shanghai, China).

\section{Neuronal culture preparation and transfections}

Dissociated neuronal cultures were prepared from postnatal day 0 (P0) Sprague-Dawley rat pups as described previously [85, 86]. Briefly, hippocampi were dissected (dentate gyri removed), dissociated and plated onto PDL (P6407, Sigma-Aldrich, St. Louis, MO, USA) coated glass coverslips (63-3009, Assistent, Sondheim/Rhön, Germany) at 15000 cells $/ \mathrm{cm}^{2}$ in Neurobasal medium (10888-022, Thermo Fisher Scientific, Waltham, MA, USA) containing $2 \mathrm{mM}$ Glutamax-I (35050-061, Thermo Fisher Scientific), B-27 (17504-044, Thermo Fisher Scientific) and 2.5\% FBS (SH30070.03, GE Healthcare Life Sciences, Pittsburgh, PA, USA). For analysis of PI subcellular localization and early neuronal development, neurons were fixed 3-6 $\mathrm{h}$ after plating.

For the analysis of axonal arbors, neurons were electroporated using Amaxa Nucleofactor Device II (Lonza, Valais, Switzerland), plated at 50000 cells $/ \mathrm{cm}^{2}$ onto matrigel (354234, BD Biosciences, Sparks, MD, USA) coated glass coverslips and fixed at DIV 3. For the analysis of primary dendrites, neurons were plated at 50000 cells $/ \mathrm{cm}^{2}$, transfected using calcium phosphate at DIV $2(2-4 \mu \mathrm{g}$ DNA per 24-well) and fixed for analysis at DIV 5.

\section{In utero electroporation}

In utero electroporation was carried out as previously described [23]. Briefly, timed-pregnant Sprague-Dawley rats were anesthetized using $12 \%$ chloral hydrate at $3.5 \mathrm{ml} / \mathrm{kg}$. Lateral ventricles of E15.5 embryos were injected with $2 \mu$ l DNA, with forcep-type electrode paddles controlled by an ECM 830 electroporator (BTX Instrument Division, Harvard Apparatus Inc., Holliston, MA, USA) delivering $5 \times 50 \mathrm{~ms}$ pulses of $60 \mathrm{mV}$ at $100 \mathrm{~ms}$ intervals. Embryos were fixed at E18.5 in 4\% paraformaldehyde, and coronal cortical sections of $50 \mu \mathrm{m}$ were cut on a CM1900 cryostat (Leica Biosystems, Nussloch, Germany).

For examining the effects of SHIP2 and PI3K C2 $\alpha$ RNAi on early neuronal development, cortical cultures were prepared from E18.5 in utero electroporated embryos.

\section{Pharmacological treatments}

The following pharmacological agents (all dissolved in DMSO) were used at the specified concentrations: AS1949490, $70 \mu \mathrm{M}$ (3718, R\&D Systems, Minneapolis, MN, USA); $m$-3M3FBS, 100 $\mathrm{M}$ (1941, R\&D Systems); LY 294002, $100 \mu \mathrm{M}$ (440204, Merck, Darmstadt, Germany); Jasplakinolide, $0.1 \mu \mathrm{M}$ (J7473, Thermo Fisher Scientific); wiskostatin, $1 \mu \mathrm{g} / \mathrm{ml}$ (w2270, Sigma-Aldrich) and wortmannin (1232, R\&D Systems). 
Preparation of $\mathrm{PIP}_{2^{-}}$or $\mathrm{PIP}_{3}$-coated beads

Glass beads of 3-10 $\mu \mathrm{m}$ (07666-1, Polysciences, Warrington, PA, USA) were coated with PI(3,4) $\mathrm{P}_{2}$ (P-9034, Echelon Biosciences, Salt Lake City, UT, USA) or PIP ${ }_{3}$ (P-9039, Echelon Biosciences), using previously described protocols for coating small molecules onto beads [87]. Briefly, beads were washed in $70 \%$ ethanol for $30 \mathrm{~min}$, dried, coated with $0.5 \mathrm{mg} / \mathrm{ml}$ poly-D-lysine (P6407, Sigma-Aldrich) for $4 \mathrm{~h}$, thoroughly washed and dried. Previous work has shown that the polybasic protein histone $\mathrm{H} 1$ functioned as a carrier to rapidly and efficiently deliver PIs into intracellular compartments [44]. Following the manufacturer's instructions, the histone $\mathrm{H} 1$ carrier and $\mathrm{PI}(3,4) \mathrm{P}_{2} / \mathrm{PIP}_{3}$ were each diluted to $1 \mathrm{mM}$, and mixed for $20 \mathrm{~min}$ at room temperature in a 1:1 ratio. Glass beads were then incubated with the PI mixture overnight at $4{ }^{\circ} \mathrm{C}$ and washed twice with PBS before use. Coated beads were added to the neuronal culture at the time of plating. Successful coating was verified using fluorescently (BODIPY FL) labeled PI.

\section{DNA constructs}

The rat SHIP2 (gene name: INPPL1) RNAi construct target the

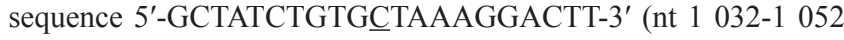
of rat INPPL1 gene; the same sequence as previously described for mouse INPPL1 [88], except for underlined nucleotide). The rat PI3K C2 $\alpha$ (gene name: PIK3 C2A) RNAi construct target the sequence 5'-GGATCTTTTTAAGCCTATT-3' (nt 2 447-2 465 of rat $P I K 3 C 2 A$; the same sequence as previously described for human PIK3 C2A except for the underlined nucleotide [57]). The Lamellipodin (Lpd; gene name: RAPH1) RNAi constructs target previously described sequences as follows: 5'-AGAAGTGCTGTTAGAGGAATG-3' (nt $1345-1365$ of rat RAPH1 gene) [56] for Lpd RNAi 1; 5'-AAGCGCCAAGTCACAGAACCC-3' (nt 524544 of rat $R A P H 1$ gene) [62] for Lpd RNAi 2; and 5'-CCCAAAGTACTGTGAGCTCTA-3' (nt 1 908-1 928 of rat RAPH1 gene) [63] for Lpd RNAi 3. The Arp3 RNAi construct was as previously described [85]. All sequences were cloned into pSuper-GFP [85].

The full-length human INPPL1 [59] (gift of Prof Alexander Sorisky, University of Ottawa, Canada) was subcloned into pCAGGS; the human sequence is different from the rat sequence at $7 \mathrm{nt}$ and thus resistant to rat SHIP2 RNAi. Seven silent mutations (underlined: 5'-AGACCTATTCAAACCAATA-3') were generated in the human PIK3 C2A sequence [57] (gift of Dr Volker Haucke, Leibniz Institut für Molekulare Pharmakologie, Berlin, Germany) to make it RNAi resistant. SHIP2 PDR/AAA [59], encoding 5'-phosphase-defective SHIP2, contains in-frame mutations P686A, D690A and R691A (gift of Prof Alexander Sorisky). The kinase-inactive mutant of PI3K C $2 \alpha$ (PI3K C $2 \alpha \mathrm{KD}$ ) was generated by mutating the ATP-binding site (K1138A, D1157A) and the catalytic loop (D1250A), as previously described [57]. The INPP4B-CAAX [57] construct was a gift of Prof Volker Haucke.

The pCS2-Arp3-expressing human Arp3 [85] is resistant to rat Arp3 RNAi. The full-length rat WASL (encoding N-WASP) [89] was a gift of Prof Marc Krischner (Harvard University, USA). N-WASP DN was generated by subcloning the first 391 amino acids of N-WASP, followed by an HA tag, into pCAGGs. For the generation of N-WASP CA, amino acids 392-501 of N-WASP were subcloned into pCAGGs immediately after the CD8 transmembrane domain [90]. To generate GST-N-WASP B motif protein, amino acids $178-244$ of N-WASP were subcloned into pGEX-KG and fused in frame with the GST tag. Stinger [73], the
N-WASP activity probe, was a gift of Prof Jane Y Wu (Northwestern University, USA).

\section{Antibodies}

Immunocytochemisty [90] and immunohistochemistry [91] were carried out as previously described. The following primary antibodies were used: $\mathrm{PI}(3,4) \mathrm{P}_{2}$ (Z-P034b, 1:200; Echelon Biosciences), $\mathrm{PI}(4,5) \mathrm{P}_{2}$ (Z-P045, 1:200; Echelon Biosciences), PI(3,4,5) $\mathrm{P}_{2}$ (Z-P345, 1:200; Echelon Biosciences), Tuj 1 (G7121, 1:5 000; Promega, Madison, WI, USA), MAP2 (AB5622, 1:1 000; Millipore, Billerica, MA, USA), SHIP2 (sc-14502, 1:500; Santa Cruz, Dallas, TX, USA) and PI3K C2 $\alpha$ (22028-1-AP, 1:500; Proteintech, Rosemont, IL, USA). Alexa Fluor 488, 568 or 633 secondary antibodies (Thermo Fisher Scientific), and Alexa Fluor 488 and 568 Phalloidin (Thermo Fisher Scientific) were used at 1:1 000. TOPRO-3 (T3605, 1:3 000; Thermo Fisher Scientific) or DAPI (D1306, 1:5 000; Thermo Fisher Scientific) was used to visualize the nuclei.

\section{Image acquisition and analyses}

Confocal images were acquired on one of the following confocal microscopes: LSM5 (Carl Zeiss, Oberkochen, Germany) with a 40 oil immersion Neofluor objective $(\mathrm{NA}=1.3)$ or $63 \times$ oil immersion Plan-Apochromat objective $(\mathrm{NA}=1.4)$, or Nikon A1R (Nikon, Tokyo, Japan) with a $60 \times$ Oil Plan Apo VC objective (NA $=1.4$ ). Images of brain slices were acquired on a LSM5 with a $20 \times$ Plan-Apochromat $(\mathrm{NA}=0.75)$ objective. Images of axon length were acquired on a FV10I confocal laser scanning microscope (Olympus, Tokyo, Japan) with a 60 phase-contrast oil immersion objective $(\mathrm{NA}=1.35)$. SIM was acquired on a Nikon N-SIM microscope, with a HP Apo TIRF $100 \times \mathrm{H}$ objective $(\mathrm{NA}=1.49)$ and an Andor DU-897 X-10515 camera. For each experiment, all control and experimental conditions were acquired with the same microscope and objective and at the same laser and imaging settings.

For all morphological analyses, images were coded using computer-generated random sequences during data acquisition. Images were analyzed as acquired with no post-acquisition modifications and blinded to the experimental condition. Analyses of neuronal developmental stages and neurite number [23], total dendrite length [86], TDBTN [86], average dendrite length [86] and total axon length [92] were as previously described. For measuring PI intensity, images were thresholded, and the total PI intensity of the cell body was quantitated. For co-localization assays, images were thresholded, and overlapping pixels were quantitated against total thresholded area. Fiji/ImageJ (NIH, Bethesda, MD, USA) was used to quantitate the intensity of PI and F-actin across the cell body. Only protrusions immune-positive for Tuj 1 and at least $2 \mu \mathrm{m}$ in length were considered to be neurites. Axonal and dendritic arbors were traced using ImageProPlus (Media-Cybernetics, Rockville, MD, USA). N-SIM images were 3D reconstructed using N-SIM analysis software. Example images were adjusted for brightness/contrast within linear ranges using Fiji/ImageJ when necessary. Control and experiment conditions were adjusted with the same parameters.

\section{FRET}

Neurons were electroporated at the time of plating and imaged at DIV 5 using a Nikon A1R microscope and a Plan Apo VC 60× Oil DIC N2 objective (NA = 1.4). Carrier (control) or carrier and 
$\mathrm{PI}(3,4) \mathrm{P}_{2}(20 \mu \mathrm{M})$ was added to the culture medium for $1 \mathrm{~h}$ and washed out immediately before the start of the imaging session. Dual-emission intensity ratios were recorded; filter combinations were as follows (excitation:emission): $\mathrm{CFP}: \mathrm{CFP}(403,480)$ and CFP:YFP $(403,540)$. The YFP/CFP ratio was measured along a 5 $\mu \mathrm{m}$ stretch close to the cell membrane and calculated using original images in Fuji/ImageJ. Example images shown in Figure 8B were smoothed $(3 \times 3$ filter $)$.

\section{Immunoprecipitation and western blot}

GST and GST-N-WASP B motif were expressed in E. coli strain BL21 (DE3) and induced using $0.1 \mathrm{mM} \mathrm{IPTG}$ at $16{ }^{\circ} \mathrm{C}$ overnight. Proteins were purified using GST Spin Purification kit (16106, Thermo Fisher Scientific) in the presence of protease inhibitor cocktail tablets (04693116001, Roche Diagnostics, Indianapolis, IN, USA) and according to the manufacturer's protocols. Control lipid beads (P-B000, Echelon Biosciences) and $\mathrm{PI}(3,4) \mathrm{P}_{2}$ beads (P-B034a, Echelon Biosciences) were incubated with $2 \mu \mathrm{g}$ purified proteins overnight at $4{ }^{\circ} \mathrm{C}$, washed five times and resuspended in $100 \mu 1$ sample buffer for western blot analysis. Western blot was performed according to the standard protocols, with the following primary antibodies: GST (66001-1, 1:2 000; Proteintech), SHIP2 (sc-14502, 1:500; Santa Cruz), PI3K C2 $\alpha$ (22028-1AP, 1:500; Proteintech) and GAPDH (KC-5G4, 1:10 000; KangChen Bio-tech, SH, China).

\section{Statistics}

Statistical analyses were performed in GraphPad Prism 5 (GraphPad Software, La Jolla, CA, USA) using Student's $t$-test (for sample pairs), one-way ANOVA (for three or more samples) followed by Tukey's or Dunnett's multiple comparisons test, or twoway ANOVA (for comparing two independent variables) followed by Bonferroni's multiple comparisons test, as indicated in the figure legends. Results are shown as mean \pm SEM, and $n$ refers to the number of cells unless otherwise stated. All conditions statistically different from controls are indicated: ${ }^{*} P<0.05 ; * * P<0.01$; $* * * P$ $<0.001$; n.s., not significant.

\section{Acknowledgments}

We thank Professors Volker Haucke, Marc Kirschner, Alexander Sorisky and Jane Y Wu for constructs. We thank Yuan Lu and Zong-Fang Wan for excellent technical assistance. We thank Dr Qian Hu and ION Optical Imaging Core Facility for confocal microscopy and data analysis support, and Dr Min Jiang and IOBS-Nikon Biological Imaging Center for use of the N-SIM microscope. We are grateful to Drs Xueliang Zhu and Xiumin Yan, colleagues at ION, and members of the Yu laboratory for suggestions and comments. This work was supported by the National Natural Science Foundation of China (31321091 and 31530030), the Strategic Priority Research Program of the Chinese Academy of Sciences (XDB02010000) and the Science and Technology Commission of Shanghai Municipality (16XD1404800).

\section{Author Contributions}

S-X Zhang, L-H Duan and X Yu designed the study. S-X Zhang and L-H Duan performed all experiments, except for generation of some molecular tools by S-J He and protein purification by G-F Zhuang. S-X Zhang and L-H Duan analyzed all data. S-X Zhang,
L-H Duan and X Yu wrote the paper.

\section{Competing Financial Interests}

The authors declare no competing financial interests.

\section{References}

1 Craig AM, Banker G. Neuronal polarity. Annu Rev Neurosci 1994; 17:267-310.

2 Dotti CG, Sullivan CA, Banker GA. The establishment of polarity by hippocampal neurons in culture. J Neurosci 1988 ; 8:1454-1468.

3 Arimura N, Kaibuchi K. Neuronal polarity: from extracellular signals to intracellular mechanisms. Nat Rev Neurosci 2007; 8:194-205.

4 Barnes AP, Polleux F. Establishment of axon-dendrite polarity in developing neurons. Annu Rev Neurosci 2009; 32:347-381.

5 Cheng PL, Poo MM. Early events in axon/dendrite polarization. Annu Rev Neurosci 2012; 35:181-201.

6 Stiess M, Bradke F. Neuronal polarization: the cytoskeleton leads the way. Dev Neurobiol 2011; 71:430-444.

7 Arikkath J. Molecular mechanisms of dendrite morphogenesis. Front Cell Neurosci 2012; 6:61.

8 Puram SV, Bonni A. Cell-intrinsic drivers of dendrite morphogenesis. Development 2013; 140:4657-4671.

9 Urbanska M, Blazejczyk M, Jaworski J. Molecular basis of dendritic arborization. Acta Neurobiol Exp 2008; 68:264-288.

10 Jan YN, Jan LY. Branching out: mechanisms of dendritic arborization. Nat Rev Neurosci 2010; 11:316-328.

11 McAllister AK. Dynamic aspects of CNS synapse formation. Annu Rev Neurosci 2007; 30:425-450.

12 Shen K, Scheiffele P. Genetics and cell biology of building specific synaptic connectivity. Annu Rev Neurosci 2010; 33:473-507.

13 Yuste R, Bonhoeffer T. Genesis of dendritic spines: insights from ultrastructural and imaging studies. Nat Rev Neurosci 2004; 5:24-34.

14 da Silva JS, Dotti CG. Breaking the neuronal sphere: regulation of the actin cytoskeleton in neuritogenesis. Nat Rev Neurosci 2002; 3:694-704.

15 Flynn KC. The cytoskeleton and neurite initiation. Bioarchitecture 2013 ; 3:86-109.

16 Sainath R, Gallo G. Cytoskeletal and signaling mechanisms of neurite formation. Cell Tissue Res 2015; 359:267-278.

17 Gallo G. Mechanisms underlying the initiation and dynamics of neuronal filopodia: from neurite formation to synaptogenesis. Int Rev Cell Mol Biol 2013; 301:95-156.

18 Gupton SL, Gertler FB. Filopodia: the fingers that do the walking. Sci STKE 2007; 2007:re5.

19 Mattila PK, Lappalainen P. Filopodia: molecular architecture and cellular functions. Nat Rev Mol Cell Biol 2008; 9:446-454.

20 Dent EW, Kwiatkowski AV, Mebane LM, et al. Filopodia are required for cortical neurite initiation. Nat Cell Biol 2007; 9:1347-1359.

21 Kwiatkowski AV, Rubinson DA, Dent EW, et al. Ena/VASP is required for neuritogenesis in the developing cortex. Neuron 2007; 56:441-455.

22 Flynn KC, Hellal F, Neukirchen D, et al. ADF/cofilin-mediated actin retrograde flow directs neurite formation in the devel- 
oping brain. Neuron 2012; 76:1091-1107.

23 Zhang SX, Duan LH, Qian H, Yu X. Actin aggregations mark the sites of neurite initiation. Neurosci Bull 2016; 32:1-15.

24 Polleux F, Morrow T, Ghosh A. Semaphorin 3A is a chemoattractant for cortical apical dendrites. Nature 2000; 404:567573.

25 Shelly M, Cancedda L, Heilshorn S, Sumbre G, Poo MM. LKB1/STRAD promotes axon initiation during neuronal polarization. Cell 2007; 129:565-577.

26 McAllister AK, Katz LC, Lo DC. Opposing roles for endogenous BDNF and NT-3 in regulating cortical dendritic growth. Neuron 1997; 18:767-778.

27 Gartner A, Fornasiero EF, Munck S, et al. N-cadherin specifies first asymmetry in developing neurons. EMBO J 2012; 31:1893-1903.

28 Stuart G, Sprouston N, Hausser M. Dendrites. 2nd Edition. Oxford: Oxford University Press, 2008.

29 Di Paolo G, De Camilli P. Phosphoinositides in cell regulation and membrane dynamics. Nature 2006; 443:651-657.

30 Suetsugu S, Kurisu S, Takenawa T. Dynamic shaping of cellular membranes by phospholipids and membrane-deforming proteins. Physiol Rev 2014; 94:1219-1248.

31 Saarikangas J, Zhao H, Lappalainen P. Regulation of the actin cytoskeleton-plasma membrane interplay by phosphoinositides. Physiol Rev 2010; 90:259-289.

32 Balla T. Phosphoinositides: tiny lipids with giant impact on cell regulation. Physiol Rev 2013; 93:1019-1137.

33 Skwarek LC, Boulianne GL. Great expectations for PIP: phosphoinositides as regulators of signaling during development and disease. Dev Cell 2009; 16:12-20.

34 Li H, Marshall AJ. Phosphatidylinositol $(3,4)$ bisphosphate-specific phosphatases and effector proteins: a distinct branch of PI3K signaling. Cell Signal 2015; 27:1789-1798.

35 Shi SH, Jan LY, Jan YN. Hippocampal neuronal polarity specified by spatially localized $\mathrm{mPar} 3 / \mathrm{mPar} 6$ and PI 3-kinase activity. Cell 2003; 112:63-75.

36 Jiang H, Guo W, Liang X, Rao Y. Both the establishment and the maintenance of neuronal polarity require active mechanisms: critical roles of GSK-3beta and its upstream regulators. Cell 2005; 120:123-135.

37 Menager C, Arimura N, Fukata Y, Kaibuchi K. PIP3 is involved in neuronal polarization and axon formation. J Neurochem 2004; 89:109-118.

38 Gassama-Diagne A, Yu W, ter Beest M, et al. Phosphatidylinositol-3,4,5-trisphosphate regulates the formation of the basolateral plasma membrane in epithelial cells. Nat Cell Biol 2006; 8:963-970.

39 Liu H, Radisky DC, Wang F, Bissell MJ. Polarity and proliferation are controlled by distinct signaling pathways downstream of PI3-kinase in breast epithelial tumor cells. $J$ Cell Biol 2004; 164:603-612.

40 Martin-Belmonte F, Gassama A, Datta A, et al. PTEN-mediated apical segregation of phosphoinositides controls epithelial morphogenesis through Cdc42. Cell 2007; 128:383-397.

41 Vlahos CJ, Matter WF, Hui KY, Brown RF. A specific inhibitor of phosphatidylinositol 3-kinase, 2-(4-morpholinyl)-8phenyl-4H-1-benzopyran-4-one (LY294002). J Biol Chem 1994; 269:5241-5248.

42 Suwa A, Yamamoto T, Sawada A, et al. Discovery and func- tional characterization of a novel small molecule inhibitor of the intracellular phosphatase, SHIP2. Br J Pharmacol 2009; 158:879-887.

43 Bae YS, Lee TG, Park JC, et al. Identification of a compound that directly stimulates phospholipase $\mathrm{C}$ activity. Mol Pharmacol 2003; 63:1043-1050.

44 Ozaki S, DeWald DB, Shope JC, Chen J, Prestwich GD. Intracellular delivery of phosphoinositides and inositol phosphates using polyamine carriers. Proc Natl Acad Sci USA 2000; 97:11286-11291.

45 Gartner A, Fornasiero EF, Valtorta F, Dotti CG. Distinct temporal hierarchies in membrane and cytoskeleton dynamics precede the morphological polarization of developing neurons. J Cell Sci 2014; 127:4409-4419.

46 Shelly M, Lim BK, Cancedda L, Heilshorn SC, Gao H, Poo MM. Local and long-range reciprocal regulation of cAMP and cGMP in axon/dendrite formation. Science 2010; 327:547552 .

47 Hamilton MJ, Ho VW, Kuroda E, et al. Role of SHIP in cancer. Exp Hematol 2011; 39:2-13.

48 Muraille E, Dassesse D, Vanderwinden JM, et al. The SH2 domain-containing 5-phosphatase SHIP2 is expressed in the germinal layers of embryo and adult mouse brain: increased expression in N-CAM-deficient mice. Neuroscience 2001; 105:1019-1030.

49 Muraille E, Pesesse X, Kuntz C, Erneux C. Distribution of the src-homology-2-domain-containing inositol 5-phosphatase SHIP-2 in both non-haemopoietic and haemopoietic cells and possible involvement of SHIP-2 in negative signalling of B-cells. Biochem J 1999; 342 Pt 3:697-705.

50 Xie J, Erneux C, Pirson I. How does SHIP1/2 balance PtdIns(3,4)P2 and does it signal independently of its phosphatase activity? Bioessays 2013; 35:733-743.

51 Falasca M, Maffucci T. Regulation and cellular functions of class II phosphoinositide 3-kinases. Biochem J 2012; 443:587-601.

52 Vanhaesebroeck B, Guillermet-Guibert J, Graupera M, Bilanges $\mathrm{B}$. The emerging mechanisms of isoform-specific PI3K signalling. Nat Rev Mol Cell Biol 2010; 11:329-341.

53 Campa CC, Franco I, Hirsch E. PI3K-C2 $\alpha$ : One enzyme for two products coupling vesicle trafficking and signal transduction. FEBS Lett 2015; 589:1552-1558.

54 Yoshioka K, Yoshida K, Cui H, et al. Endothelial PI3K-C2 $\alpha$, a class II PI3K, has an essential role in angiogenesis and vascular barrier function. Nat Med 2012; 18:1560-1569.

55 Franco I, Gulluni F, Campa CC, et al. PI3K class II $\alpha$ controls spatially restricted endosomal PtdIns3P and Rab11 activation to promote primary cilium function. Dev Cell 2014; 28:647658.

56 Yoshinaga S, Ohkubo T, Sasaki S, et al. A phosphatidylinositol lipids system, lamellipodin, and Ena/VASP regulate dynamic morphology of multipolar migrating cells in the developing cerebral cortex. J Neurosci 2012; 32:11643-11656.

57 Posor Y, Eichhorn-Gruenig M, Puchkov D, et al. Spatiotemporal control of endocytosis by phosphatidylinositol-3,4-bisphosphate. Nature 2013; 499:233-237.

58 Wada T, Sasaoka T, Funaki M, et al. Overexpression of SH2-containing inositol phosphatase 2 results in negative regulation of insulin-induced metabolic actions in 3T3-L1 ad- 
ipocytes via its 5-phosphatase catalytic activity. Mol Cell Biol 2001; 21:1633-1646.

59 Artemenko Y, Gagnon A, Sorisky A. Catalytically inactive SHIP2 inhibits proliferation by attenuating PDGF signaling in 3T3-L1 preadipocytes. J Cell Physiol 2009; 218:228-236.

60 Gaidarov I, Zhao Y, Keen JH. Individual phosphoinositide 3-kinase $\mathrm{C} 2 \alpha$ domain activities independently regulate clathrin function. $J$ Biol Chem 2005; 280:40766-40772.

61 Michael M, Vehlow A, Navarro C, Krause M. c-Abl, Lamellipodin, and Ena/VASP proteins cooperate in dorsal ruffling of fibroblasts and axonal morphogenesis. Curr Biol 2010; 20:783-791.

62 Krause M, Leslie JD, Stewart M, et al. Lamellipodin, an Ena/ VASP ligand, is implicated in the regulation of lamellipodial dynamics. Dev Cell 2004; 7:571-583.

63 Carmona G, Perera U, Gillett C, et al. Lamellipodin promotes invasive $3 \mathrm{D}$ cancer cell migration via regulated interactions with Ena/VASP and SCAR/WAVE. Oncogene 2016; 35:51555169.

64 Bae YH, Ding Z, Das T, Wells A, Gertler F, Roy P. Profilin1 regulates $\mathrm{PI}(3,4) \mathrm{P} 2$ and lamellipodin accumulation at the leading edge thus influencing motility of MDA-MB-231 cells. Proc Natl Acad Sci USA 2010; 107:21547-21552.

65 Dowler S, Currie RA, Campbell DG, et al. Identification of pleckstrin-homology-domain-containing proteins with novel phosphoinositide-binding specificities. Biochem J 2000; 351:19-31.

66 Papayannopoulos V, Co C, Prehoda KE, Snapper S, Taunton J, Lim WA. A polybasic motif allows N-WASP to act as a sensor of PIP(2) density. Mol Cell 2005; 17:181-191.

67 Miki H, Miura K, Takenawa T. N-WASP, a novel actin-depolymerizing protein, regulates the cortical cytoskeletal rearrangement in a PIP2-dependent manner downstream of tyrosine kinases. EMBO J 1996; 15:5326-5335.

68 Stradal TE, Rottner K, Disanza A, Confalonieri S, Innocenti M, Scita G. Regulation of actin dynamics by WASP and WAVE family proteins. Trends Cell Biol 2004; 14:303-311.

69 Takenawa T, Suetsugu S. The WASP-WAVE protein network: connecting the membrane to the cytoskeleton. Nat Rev Mol Cell Biol 2007; 8:37-48.

70 Pollard TD, Beltzner CC. Structure and function of the Arp2/3 complex. Curr Opin Struct Biol 2002; 12:768-774.

71 Higgs HN, Pollard TD. Activation by Cdc42 and PIP(2) of Wiskott-Aldrich syndrome protein (WASp) stimulates actin nucleation by Arp2/3 complex. J Cell Biol 2000; 150:13111320.

72 Rohatgi R, Ma L, Miki H, et al. The interaction between N-WASP and the Arp2/3 complex links Cdc42-dependent signals to actin assembly. Cell 1999; 97:221-231.

73 Ward ME, Wu JY, Rao Y. Visualization of spatially and temporally regulated N-WASP activity during cytoskeletal reorganization in living cells. Proc Natl Acad Sci USA 2004; 101:970-974.

74 Peterson JR, Bickford LC, Morgan D, et al. Chemical inhibition of N-WASP by stabilization of a native autoinhibited conformation. Nat Struct Mol Biol 2004; 11:747-755.
75 Berridge MJ. Inositol trisphosphate and calcium signalling mechanisms. Biochim Biophys Acta 2009; 1793:933-940.

76 Cantley LC. The phosphoinositide 3-kinase pathway. Science 2002; 296:1655-1657.

77 Chau AH, Walter JM, Gerardin J, Tang C, Lim WA. Designing synthetic regulatory networks capable of self-organizing cell polarization. Cell 2012; 151:320-332.

78 Marshall AJ, Krahn AK, Ma K, Duronio V, Hou S. TAPP1 and TAPP2 are targets of phosphatidylinositol 3-kinase signaling in B cells: sustained plasma membrane recruitment triggered by the B-cell antigen receptor. Mol Cell Biol 2002; 22:5479-5491.

79 Oikawa T, Itoh T, Takenawa T. Sequential signals toward podosome formation in NIH-src cells. J Cell Biol 2008; 182:157-169.

80 Threadgill R, Bobb K, Ghosh A. Regulation of dendritic growth and remodeling by Rho, Rac, and Cdc42. Neuron 1997; 19:625-634.

81 Jaffe AB, Hall A. Rho GTPases: biochemistry and biology. Annu Rev Cell Dev Biol 2005; 21:247-269.

82 Newey SE, Velamoor V, Govek EE, Van Aelst L. Rho GTPases, dendritic structure, and mental retardation. J Neurobiol 2005; 64:58-74.

83 Luo L. Actin cytoskeleton regulation in neuronal morphogenesis and structural plasticity. Annu Rev Cell Dev Biol 2002; 18:601-635.

84 Verhage M, Maia AS, Plomp JJ, et al. Synaptic assembly of the brain in the absence of neurotransmitter secretion. Science 2000; 287:864-869.

85 Tan ZJ, Peng Y, Song HL, Zheng JJ, Yu X. N-cadherin-dependent neuron-neuron interaction is required for the maintenance of activity-induced dendrite growth. Proc Natl Acad Sci USA 2010; 107:9873-9878.

$86 \mathrm{Yu} \mathrm{X,} \mathrm{Malenka} \mathrm{RC.} \beta$-catenin is critical for dendritic morphogenesis. Nat Neurosci 2003; 6:1169-1177.

87 Shelly M, Lim BK, Cancedda L, Heilshorn SC, Gao HF, Poo MM. Local and long-range reciprocal regulation of cAMP and cGMP in axon/dendrite formation. Science 2010; 327:547552.

88 Yoshinaga S, Ohkubo T, Sasaki S, et al. A phosphatidylinositol lipids system, lamellipodin, and Ena/VASP regulate dynamic morphology of multipolar migrating cells in the developing cerebral cortex. J Neurosci 2012; 32:11643-11656.

89 Ho HY, Rohatgi R, Lebensohn AM, Kirschner MW. In vitro reconstitution of cdc42-mediated actin assembly using purified components. Methods Enzymol 2006; 406:174-190.

90 Bian WJ, Miao WY, He SJ, Wan ZF, Luo ZG, Yu X. A novel Wnt5a-Frizzled4 signaling pathway mediates activity-independent dendrite morphogenesis via the distal PDZ motif of Frizzled 4. Dev Neurobiol 2015; 75:805-822.

91 Zheng JJ, Li SJ, Zhang XD, et al. Oxytocin mediates early experience-dependent cross-modal plasticity in the sensory cortices. Nat Neurosci 2014; 17:391-399.

$92 \mathrm{Yu}$ X, Malenka RC. Multiple functions for the cadherin/catenin complex during neuronal development. Neuropharmacology 2004; 47:779-786.

(Supplementary information is linked to the online version of the paper on the Cell Research website.) 